\title{
Pre-processing, group accretion and the orbital trajectories of associated subhaloes
}

\author{
Lucie Bakels $^{1,2, \star}$, Aaron D. Ludlow ${ }^{1,2}$ and Chris Power ${ }^{1,2}$ \\ ${ }^{1}$ International Centre for Radio Astronomy Research, University of Western Australia, 35 Stirling Highway, Crawley, \\ Western Australia, 6009, Australia \\ ${ }^{2}$ ARC Centre of Excellence for All-Sky Astrophysics in $3 D$ (ASTRO 3D)
}

8 January 2021

\begin{abstract}
We use a high-resolution cosmological dark matter-only simulation to study the orbital trajectories of haloes and subhaloes in the environs of isolated hosts. We carefully tally all apsis points and use them to distinguish haloes that are infalling for the first time from those that occupy more evolved orbits. We find that roughly 21 per cent of resolved subhaloes within a host's virial radius are currently on first infall, and have not yet reached their first orbital pericentre; roughly 44 per cent are still approaching their first apocentre after infall. For the range of host masses studied, roughly half of all accreted systems were pre-processed prior to infall, and about 20 per cent were accreted in groups. We confirm that the entire population of accreted subhaloes - often referred to as "associated" subhaloes - extends far beyond the virial radii of their hosts, with roughly half currently residing at distances that exceed $\approx$ $1.2 \times r_{200}$. Many of these backsplash haloes have gained orbital energy since infall, and occupy extreme orbits that carry them well past their initial turnaround radii. Such extreme orbits are created during the initial accretion and dissolution of loosely bound groups, but also through penetrating encounters between subhaloes on subsequent orbits. The same processes may also give rise to unexpectedly abrupt losses of orbital energy. These effects combine, giving rise to a large variation in the ratio of sequent apocentres for accreted systems. We find that, within 2 virial radii from host centres, the concentrations of first-infall haloes are remarkably similar those of isolated field haloes, whereas backsplash haloes, as well as systems that were pre-processed, are considerably more concentrated.
\end{abstract}

Key words: cosmology: dark matter - methods: numerical - galaxies: formation, evolution

\section{INTRODUCTION}

In the standard cosmological model the variance of linear matter density fluctuations increases systematically toward smaller scales. The dominant mass component is a cold and collisionless particle - referred to as cold dark matter (CDM hereafter) - and the energy density of the Universe is dominated by dark energy (denoted $\Lambda$ in the simplest case of a cosmological constant). This is the $\Lambda$ CDM model, which has few rivals in its ability to accurately describe the large( $\gtrsim 10 \mathrm{Mpc}$ ) and intermediate-scale $(1-10 \mathrm{Mpc})$ structure of the Universe (see Frenk \& White 2012, for a recent review).

$\Lambda \mathrm{CDM}$ also makes a few unwavering predictions. One,

* E-mail: lucie.bakels@research.uwa.edu.au a consequence of the shape of the density fluctuation power spectrum, is that structure formation proceeds hierarchically, from small to large scales. Dark matter accretes onto primordial overdensities, either smoothly or through mergers, which grow progressively more massive over time, forming gravitationally-bound dark matter haloes. The vestiges of a halo's past accretion or merger events form a population of "substructure" haloes, or subhaloes for short. Haloes are the likely sites of galaxy formation (e.g. White \& Rees 1978), and subhaloes the potential hosts of satellite galaxies, such as those observed around the Milky Way or other nearby galaxies, or of the individual galaxies in rich clusters. The dynamics, spatial distribution and structure of substructure haloes therefore hold valuable clues to the hierarchical nature of galaxy formation.

There is now a broad and comprehensive literature ad- 
Bakels et al.

dressing a variety of issues related to the structure and substructure of dark matter haloes. For example, it is now known that substructure makes an important but subdominant contribution to the total mass of a halo, typically $\lesssim 10-15$ per cent (Ghigna et al. 1998; Neto et al. 2007), and that their mass function is well-approximated by a single power-law, $d \mathrm{~N} / d \log \mathrm{M} \propto \mathrm{M}^{-0.9}$ (Springel et al. 2001, Gao et al. 2004, Springel et al. 2008 Giocoli et al. 2010 Garrison-Kimmel et al. 2014). Subhaloes are spatially (e.g. De Lucia et al. 2004 Springel et al. 2008, Gao et al. 2012 ) and kinematically (e.g. Gill et al. 2004; Diemand et al. 2004 Sales et al. 2007b Ludlow et al. 2009) biased with respect to the underlying distribution of dark matter, which likely indicates that different environmental process shape the orbital evolution of substructure compared to smoothlyaccreted dark matter.

As with dark matter haloes, subhaloes can have substructure of their own, a hierarchy that can, in principle, extend to the free-streaming limit of the dark matter particle (indeed, four nested levels of subhaloes-in-subhaloes have been observed in highest-resolution simulation of the Aquarius Project; Springel et al. 2008). As a result, many subhaloes will have been be accreted as part of a substructure group (e.g. Li \& Helmi 2008), or will have been pre-processed in some manner prior to accretion (Wetzel et al. 2015, Han et al. 2018; Bahé et al. 2019).

In addition, when a halo or group of haloes is accreted by a more massive system, it will not necessarily remain a subhalo indefinitely. Instead, accreted haloes can pass through temporary phases of being nominal substructures - i.e. confined to the virial boundaries of their host haloes and pass again into the field, reaching orbital radii that can, in principle, extend to many times the host's virial radius (e.g. Gill et al. 2005 Bahé et al. 2013 Haggar et al. 2020). As a result, galaxies in the field may show tell-tale signs of having passed through the dense central regions of more massive systems. Indeed, simulations suggest that as many as half of all accreted systems currently lie at distances that exceed the traditional virial boundaries of their hosts (Ludlow et al. 2009). These are often referred to as "backsplash", or "associated" subhaloes, terms we adopt in this paper.

This can have important consequences. For example, at fixed luminosity, galaxies hosted by backsplash haloes have higher mass-to-light ratios (Knebe et al. 2011) and higher quenched fractions (Simpson et al. 2018) than field galaxies. The mass profiles of backsplash haloes are more concentrated than those of field haloes at comparable radial separations from their hosts, at least partially explaining a puzzling phenomenon known as assembly bias (Wang et al. 2009a, Li et al. 2013, Sunayama et al. 2016, Mansfield \& Kravtsov 2020).

The accretion of groups of substructure expected in $\Lambda \mathrm{CDM}$ also has important consequences. One is that accreted groups, when interacting with their host haloes near orbital pericentre, can lead to multi-body interactions, a scenario favourable for the rapid exchange of orbital energy and angular momentum between group members. This can, in principle, result in the ejection of (typically low-mass) subhaloes on highly "unorthodox" orbits (Sales et al.|2007a), which often propel them to distances that exceed their nominal turnaround radius (Ludlow et al. 2009). As we will see in Section 3.5, the same interactions can also lead to an abrupt loss of orbital energy and angular momentum, confining subhaloes to the innermost regions of their host haloes.

One consequence of group accretion, mentioned above, is that many systems, apparently infalling for the first time, will have been "pre-processed" prior to accretion (i.e. will have been a subhalo of a more massive halo prior infall). Simulation work suggests that as many as half of the Milky Way's satellites (with $\mathrm{M}_{\star} \gtrsim 10^{6} \mathrm{M}_{\odot}$ ) may have experienced such pre-processing (Wetzel et al. 2015), and likely more in galaxy groups and clusters. Bahé et al. (2019), for example, found that as many as 87 per cent of $\gtrsim 10^{10} \mathrm{M}_{\odot}$ haloes accreted by massive clusters were pre-processed, and 73 per cent of Milky Way-mass $\left(\sim 10^{12} \mathrm{M}_{\odot}\right)$ haloes were. In group environments, these numbers are $\approx 70$ per cent and 35 per cent, respectively.

There is also observational evidence for pre-processing. Group catalogues based on the Sloan Digital Sky Survey (data-release 7; Yang et al. 2007) have been used to classify galaxies as infall or backsplash, central or satellite. In the radial range between 2 to 3 virial radii from hosts, satellite galaxies show a higher quenched fraction than centrals, even when controlling for backsplash galaxies (Hou et al. 2014). Results from the Local Cluster Substructure Survey (LoCuSS; Bianconi et al. 2018) suggest that groups of galaxies infalling onto clusters possess lower star formation rates, on average, than the total infalling population. These are only a couple of examples of the rich literature exposing pre-processing as an important driver of galaxy evolution (see also, e.g., Cortese et al. 2006, Wetzel et al. 2014. Just et al. 2019).

Our goal is to reassess some of these issues, aided by high-resolution cosmological (dark matter-only) simulations. We focus our analysis on well-resolved and isolated primary haloes, and consider all secondary haloes (whether subhalo or field halo) that ever come with 4 virial radii of their host. We carefully classify the trajectories of secondary haloes using sensible diagnostics based on their orbital histories. Rather than drawing arbitrary distinctions between infalling and accreted systems, we instead separate them based on the number of periapsis points, $N_{\text {peri }}$, measured along their trajectories: orbits for which $N_{\text {peri }}=0$ are likely infalling for the first time; $N_{\text {peri }} \geqslant 1$ represent more evolved orbits. As discussed below, many infalling haloes have been pre-processed, a result that has important implications for not only their internal structure, but also for the ensuing evolution of their orbital trajectories

Our paper is organized as follows. We describe our $\mathrm{Nu}-$ merical methods in Section 2, our simulations are described in Section 2.1. our halo-finding techniques in Section 2.2. our merger trees and orbit tracking methods in Sections 2.3 and 2.4 respectively. In Section 3 we describe our main findings: Section 3.1 focuses on the spatial distribution of associated and first-infall haloes and subhaloes; the distribution and evolution of their apsis points are described in Sections 3.2 and 3.3 respectively. The importance of preprocessing and group infall relative to the accretion of "pristine" field haloes is discussed in Section 3.4 and the corresponding impact on subsequent dynamics in Section 3.5 Finally, in Section 3.6. we comment on the importance of pre-processing and group-infall for the structural scaling relations of dark matter haloes and subhaloes in the vicinity of massive systems. We summarize our results in Section 4 


\section{SIMULATIONS AND ANALYSIS}

\subsection{Simulations}

Our results are based on a high-resolution, cosmological dark-matter only simulation carried out with a lean version of GADGET-2 (Springel 2005). The simulation is part of the Genesis Simulations suite of cosmological $N$-body runs (cf. Poulton et al. 2019), and follows the evolution of $\mathrm{N}_{\mathrm{DM}}=$ $2048^{3}$ collisionless dark matter particles in a cubic box of comoving side-length $L=105 h^{-1} \mathrm{Mpc}$. The softening length, fixed in comoving units, is $\epsilon=1.7 h^{-1} \mathrm{kpc}$ (roughly $1 / 30^{t h}$ of the Lagrangian mean inter-particle spacing); the particle mass is $m_{p}=1.17 \times 10^{7} h^{-1} \mathrm{M}_{\odot}$. Particle data are saved as snapshots at 190 discrete intervals between $z=20$ and 0 , equally spaced in the natural logarithm of the expansion factor, which allows us to robustly track the orbits and assembly histories of haloes and subhaloes.

Initial conditions were created at $z=99$ by perturbing an initially uniform particle lattice using secondorder Lagrangian perturbation theory (Scoccimarro 1998 Crocce et al. 2006) in accord with the linear power spectrum determined by the Planck Collaboration (2016). The cosmological density of baryons, matter and dark energy are $\left(\Omega_{\mathrm{bar}}, \Omega_{\mathrm{M}}, \Omega_{\Lambda}\right)=(0.0491,0.3121,0.6879) ; \mathrm{H}_{0}=$ $67.5 \mathrm{~km} / \mathrm{s} / \mathrm{Mpc}$ is the Hubble-Lemaitre constant; $\sigma_{8}=0.815$ is the linear rms density fluctuation in $8 \mathrm{Mpc}$ spheres; $n_{s}=0.965$ is the spectral index of primordial power-law density perturbations.

\subsection{Halo identification and selection}

Haloes and subhaloes are identified using VELOCIRAPTOR (Elahi et al. 2019), which operates in two stages. First, distinct haloes are identified using a friends-of-friends (FOF) algorithm (e.g. Davis et al. 1985) employing a linking length of $b=0.2$ times the mean inter-particle spacing. Substructure haloes are then excised from each FOF group by searching for dynamically-distinct particle subsets in sixdimensional phase-space (i.e. using a 6DFOF algorithm), and correspond to local overdensities whose velocity distributions differ substantially from that of the locally-averaged background halo. Following van den Bosch (2017) and van den Bosch \& Jiang (2016), haloes and subhaloes resolved with fewer than 50 particles (at $z=0$ ) are also discarded. Their centres coincide with the coordinate of the particle with the minimum potential energy, and their bulk velocities are defined using the centre-of-mass velocity of all particles in the $6 \mathrm{DFOF}$ envelope.

For FOF haloes, VELOCIRAPTOR calculates various commonly-used definitions of virial mass. For our analysis we adopt $\mathrm{M}_{200}$, the mass contained within a sphere of radius $r_{200}$ that encloses a mean density of $200 \times \rho_{\text {crit }}$ ( $\rho_{\text {crit }}=3 H^{2} / 8 \pi G$ is the critical density for a closed universe). For substructure, masses are defined using the full subset of particles that VELOCIRAPTOR deems dynamically associated (i.e. the 6DFOF mass). In addition to mass, we also calculate the radius $R_{\max }$ at which the circular velocity profile reaches its maximum value, $V_{\max }-$ we use these as non-parametric measures of the internal structure of haloes and subhaloes.

For our analysis, we identify a sample of 2309 primary haloes with virial masses $\mathrm{M}_{200} \geqslant 10^{12} h^{-1} \mathrm{M}_{\odot}\left(\mathrm{N}_{200} \gtrsim 8.6 \times\right.$ $10^{4}$ ) that also satisfy two isolation criteria: one ensures that it is the most massive system within eight times its virial radius, and the other that the host is not within eight virial radii of a more massive system. We hereafter refer to these as primary haloes. The most massive primary in our sample has a virial mass of $\mathrm{M}_{200}=3.36 \times 10^{14} h^{-1} \mathrm{M}_{\odot}\left(\mathrm{N}_{200} \approx\right.$ $\left.2.9 \times 10^{7}\right)$.

Following custom, we refer to substructure haloes that lie within one virial radius $r_{200}$ of any primary host as "primary subhaloes". Isolated "field" haloes that lie beyond $r_{200}$ of primary hosts and have never been substructures of a more massive system are referred to as secondary haloes (see Section 2.4 for details).

\subsection{Merger trees}

Halo and subhalo merger trees are constructed using TreeFrog (see Elahi et al. 2018, 2019, for details), which is part of the VELOCIRAPTOR software package. Moving forward (from high- to low- $z$ ) through the list of halo catalogues, TreeFrog links haloes identified in snapshot $S_{i}$ to their most probable descendant in snapshot $S_{i+1}$ by maximising a figure-of-merit, defined

$\Psi^{2}=\frac{\mathrm{N}_{S_{i}^{k} \cap S_{i+1}^{l}}^{2}}{\mathrm{~N}_{S_{i}}^{k} \mathrm{~N}_{S_{i+1}}^{l}}$.

Here $\mathrm{N}_{S_{i}}^{k}$ is the number of particles in progenitor $k$ in snapshot $S_{i}, \mathrm{~N}_{S_{i+1}}^{l}$ are the numbers of particles in each unique descendant $l$ in snapshot $S_{i+1}$, and $\mathrm{N}_{S_{i}^{k} \cap S_{i+1}^{l}}$ are the number of particles they have in common.

Rarely, idiosyncratic cases - such as multiple mergers of approximately equal-mass systems - lead to several equallyprobable descendants being identified. These issues are dealt with following Poole et al. (2017), by weighting each descendant's figure-of-merit by the rank-ordered binding energy of particles $S^{k} \cap S_{i+1}^{l}$, where the rank ordering is carried out for particles in both the progenitor and descendant, thereby maximizing the fraction of highly-bound mass between the two. Other common failures of the algorithm arise when subhaloes temporarily disappear from the halo catalogues, either during a close passage through the centre of a more massive system, or by dropping below VELOCIRAPTOR 's 20-particle detection limit. We mend such occurrences by searching for descendants across a series of five consecutive snapshots until a suitable descendant is found; if one is not, we assume the halo to have merged with its host, or to be tidally disrupted (details of the algorithm can be found in Poulton et al. 2019).

One distinguishing feature of VELOCIRAPTOR is its ability to identify dynamically cold "substructure" that is slightly overdense with respect to the smooth background halo. A downside - at least for our purposes - is its tendency to identify subhaloes with strong tidal features and tidal debris from recently disrupted subhaloes which must be distinguished from gravitationally-bound substructure. These sorts of systems are transients in our halo catalogues and can be easily identified by imposing constraints on their merger trees. For example, we find that the vast majority are eliminated by demanding that each (sub)halo in the merger tree catalogue is able to be tracked through at least 


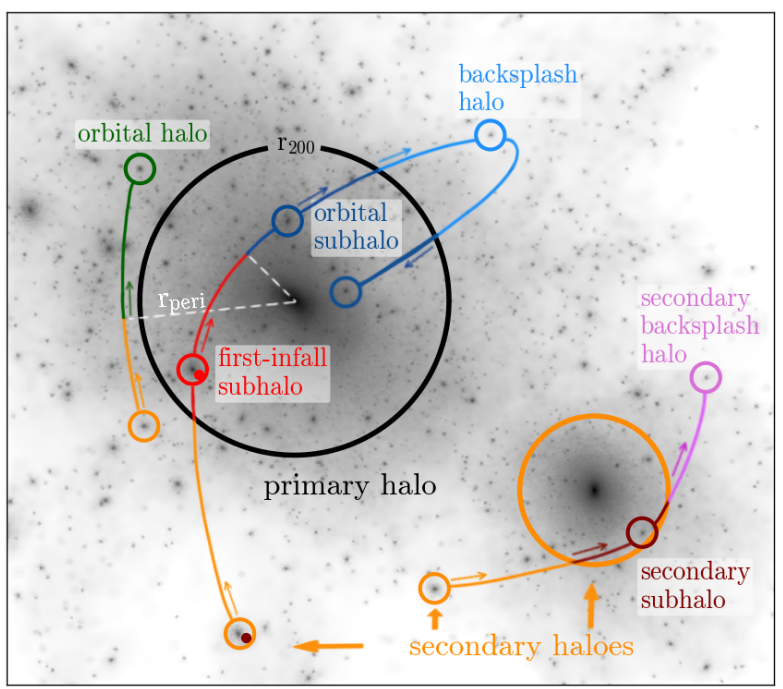

Figure 1. A visual representation of the various orbital categories defined in Section 2.4 The solid black circle marks the virial radius, $r_{200}$, of a single primary host halo; isolated secondary haloes are shown using orange circles. Upon first crossing the virial radius of the primary, a secondary halo becomes a first-infall subhalo (red), and after crossing orbital pericentre, an orbital subhalo (dark blue). Orbital subhaloes whose trajectories carry them beyond the virial radius of the primary are labelled "backsplash" haloes (light blue) as long as $r_{\text {sub }} \geqslant r_{200}$. Secondary haloes may also cross pericentre outside of their host's virial radius; these are "orbital haloes" (green). Similarly, secondary haloes may also have their own populations of secondary subhaloes (burgundy) or secondary backsplash haloes (magenta) - these define a sample of "pre-processed" systems.

8 consecutive simulation snapshots (which corresponds to about one-quarter of the circular orbital time at $r_{200}$, i.e. $\left.t_{\text {orb }} / 4=(\pi / 2) r_{200} / V_{200}\right)$.

\subsection{Subhalo orbits and their classification}

We study the dynamical and structural evolution of surviving (i.e. $z=0$ ) haloes and subhaloes that have at some point in the past crossed within $4 \times r_{200}$ of any of the primary host haloes that meet the isolation criteria described in Section 2.2. Orbits are calculated with respect to these haloes directly from the merger trees using ORBWEAVER (Poulton et al. 2019), which identifies any apsis points along a halo or subhalo's trajectory about their primary host, as well as other quantities of interest (e.g. orbital eccentricities, angular momenta, etc.).

The orbital histories of these (sub)haloes can be quite complex, and depend strongly on their interaction history with the primary halo, or on interactions with other haloes in the primary's vicinity. van den Bosch (2017), for example, identified 12 distinct evolutionary pathways through which subhaloes can evolve between simulation outputs, including frequent penetrating subhalo-subhalo encounters and subhalo exchange between distinct hosts.

Distinguishing haloes and subhaloes is non-trivial; one common approach is to differentiate them based on whether they happen to lie inside or outside their host halo's virial radius at a particular time, respectively. But as recently pointed out by Diemer (2020), this blurs the halo-subhalo distinction due to the somewhat arbitrary density thresholds used to define virial radii. These authors argue in favour adopting physically well-defined radii, such as the splashback radius (Diemer \& Kravtsov 2014), to delineate haloes and subhaloes, but this raises additional concerns. Why, for example, should any infalling halo be reclassified as a subhalo simply due to its proximity to the host?

This motivates our approach, which is primarily to divide haloes and subhaloes into distinct categories based on whether or not they have crossed orbital pericentre on their orbit about the primary host halo, without appealing to arbitrary crossing radii, like $r_{200}$. Doing so results in two distinct groups, which we now define:

Orbital haloes and subhaloes: The full population of haloes and subhaloes that have crossed pericentre at least once on an orbit defined relative to the centre-of-mass motion of their primary. These include current subhaloes of the primary halo (i.e. those with $r_{\text {sub }} \leqslant r_{200}$, provided they have crossed pericentre), the entire population of "backsplash" haloes that were once within $r_{200}$ but have since left, as well as a small fraction of haloes whose pericentres exceed the virial radius of their primary host. Orbital haloes and subhaloes can be divided into three distinct subcategories:

(i) Orbital subhaloes are currently within $r_{200}$ and have passed orbital pericentre at least once. Note that we do not restrict pericentres to radii $r_{\text {peri }}<r_{200}$ and therefore a small fraction of orbital subhaloes, $\approx 2$ per cent, are infalling for the first time (i.e. their last orbital pericentre occurred at $r>r_{200}$, but their current separation is $\left.r_{\text {sub }} \leqslant r_{200}\right)$.

(ii) Orbital haloes have periapses that exceed $r_{200}$ and have never crossed the virial radius of their host; they have never been classified as subhaloes. These haloes occupy grazing orbits, and are sometimes referred to as "fly-bys".

(iii) Backsplash haloes are seemingly isolated systems that have crossed within the virial radius of their primary host at least once in the past, but have since left $\left(r_{\text {sub }} \geqslant r_{200}\right.$ at $z=0)$.

The second distinct group of haloes and subhaloes are characterized as follows:

First-infall haloes and subhaloes: Any halo or subhalo, regardless of its radial separation from the primary, that has not yet crossed periapsis on its orbit relative to the primary. These include all haloes (and their subhaloes) at $r \geqslant r_{200}$ that are not classified as orbital (ii) or backsplash (iii) haloes, as well as subhaloes of the primary (with $r_{\text {sub }} \leqslant r_{200}$ ) that are on first approach (i.e. $\mathrm{N}_{\text {peri }}=0$ ). We define the following distinct categories of first-infall systems:

(iv) Secondary haloes are systems that have never been identified as a substructure halo of a more-massive system and have not yet crossed pericentre on their orbit relative to the primary host.

(v) Secondary subhaloes are, at $z=0$, located within the virial radius of any halo outside the virial radius, $r_{200}$, of a primary host. In practice, $\approx 93$ per cent of these are subhaloes of secondary haloes (iv); $\approx 3$ per cent are subhaloes 
of backsplash haloes (iii), and 4 per cent are subhaloes of secondary backsplash haloes (vi, below).

(vi) Secondary backsplash haloes were once secondary subhaloes, but have since veered beyond the virial boundary of their secondary host halo.

(vii) First-infall subhaloes are within the virial boundary of their primary host, but have not yet crossed first pericentre.

Figure 1 provides a visual representation of the various orbital types described above. Note that secondary haloes (iv) and subhaloes (v) only exist beyond $r_{200}$ of their primary halo; after crossing $r_{200}$ (but before reaching pericentre) we refer to them as first-infall subhaloes (vii) in order to distinguish them from orbital subhaloes (i). Secondary backsplash haloes (vi) and subhaloes (v) form a population of "pre-processed" systems that have encountered dense environments beyond the virial boundary of the primary host. Of course, any associated subhalo (a term we will often use for the combined population of orbital subhaloes (i), backsplash haloes (iii) and first-infall subhaloes (vii)) may have been pre-processed as well, and we will draw explicit distinctions between the two samples when necessary.

\section{RESULTS}

\subsection{Spatial distribution of associated subhaloes and secondary haloes}

Figure 2 plots the phase-space diagram of radial velocity versus distance for orbital and first-infall haloes and subhaloes relative to their primary hosts. Data are shown for primaries in the mass range $10^{13} \leqslant \mathrm{M}_{200} /\left[h^{-1} \mathrm{M}_{\odot}\right] \leqslant 10^{14}$, and use different colours to highlight several examples of the orbital histories outlined above. Red and dark blue points, for example, show $\left(r_{\text {sub }} \leqslant r_{200}\right)$ subhaloes of primary hosts that are either on first-infall or have crossed first pericentre within $r_{200}$ of the primary, respectively. Light blue points correspond to primary backsplash haloes. First-infall haloes and subhaloes that have not yet crosses $r_{200}$ of the primary are plotted as orange points (for clarity, we have combined categories iv, $\mathrm{v}$ and vi). A minority $(\approx 5$ per cent of all systems within $\left.4 \times r_{200}\right)$ are orbital haloes, coloured green, the majority of which $\left(\approx 94\right.$ per cent) are beyond $r_{200}$ of the primary at $z=0$.

The lower panel shows the fraction of haloes/subhaloes of each type as a function of distance from the primary. Not surprisingly, orbital subhaloes (dark blue) dominate at radii $r_{\text {sub }} \leqslant r_{200}$, making up roughly 78 per cent of classicallydefined "substructures" (i.e. of all subhaloes with $r_{\text {sub }} \leqslant$ $\left.r_{200}\right)$. First infall subhaloes (dark red) are nevertheless quite common, accounting for $\approx 24$ per cent of subhaloes at $r_{\text {sub }} \approx$ $r_{200}$, and of order 11 per cent at $r_{\text {sub }} \lesssim 0.1 \times r_{200}$, where a central galaxy is expected to dominate; approximately 22 per cent of all subhaloes within $r_{200}$ are on first infall. As previously noted (Gill et al. 2004, Ludlow et al. 2009), the population of backsplash haloes extends well beyond $r_{200}$ of the primary: roughly half populate regions that exceed $1.5 \times r_{200}$, and about $\approx 2$ per cent exceed $3 \times r_{200}$.

Another important point to note is that backsplash haloes (light blue points) tend to have a slight positive radial velocity bias relative to their host, at least for the primary

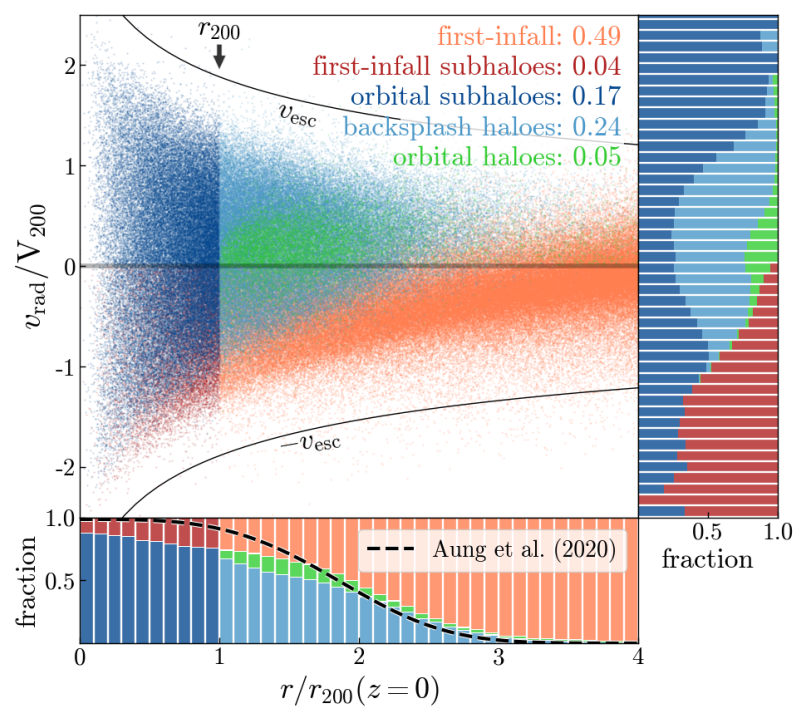

Figure 2. The main panel plots the phase-space diagram of radial velocity versus distance for haloes and subhaloes surrounding primary hosts. We combine all data for the 282 hosts that span the mass range $10^{13} \leqslant \mathrm{M}_{200} /\left[h^{-1} \mathrm{M}_{\odot}\right] \leqslant 10^{14}$ and also meet our isolation criteria (described in Section 2.2 . Coloured points differentiate the populations of (sub)haloes defined in Section 2.4 orange and red points correspond to first-infall haloes and subhaloes, respectively; blue points to systems that have completed at least one pericentric passage at $r \leqslant r_{200}$, and are currently inside (dark) or outside (light) $r_{200}$. Green points are reserved for orbital haloes - those that have completed one or more pericentric passages at radii exceeding $r_{200}$. The thin black lines demark the typical escape velocity curve for hosts in this mass range. The lower- and right-hand panels plot the fraction of (sub)haloes in each of these distinct samples that occupy discrete bins or radius or pair-wise radial velocity, respectively (for clarity, infalling haloes are left out of the latter). The black line in the bottom panel shows an estimate of the fraction of subhaloes that have completed an orbit about their hosts, as estimated by Aung et al. (2020).

mass range studied here. The mean radial velocity of all backsplash haloes, for example, is $\approx 0.28 \times \mathrm{V}_{200}$, which increases to $\approx 0.35 \times \mathrm{V}_{200}$ for those at radii $r_{\text {sub }} \gtrsim 2 \times r_{200}$. This suggests that many of these systems are still approaching first their apocentre (after turnaround), and do not have "backsplash" radii (i.e. first apocentres) that can be measured directly from their orbits (see also Diemer 2017). The right-hand panel in Figure 2 for example, shows the relative fractions of each sample in bins of radial velocity.

The number density profiles of orbital (blue curves) and first-infall haloes and subhaloes (red) are plotted in Figure 3 , for three different bins of primary halo mass (the full populations are shown using black lines). The median sphericallyaveraged density profiles of dark matter (normalized arbitrarily for comparison) are shown using grey lines. As hinted at in previous work (e.g. Diemand et al. 2004, Reed et al. 2005 Gao et al. 2004 Han et al. 2016), the spatial distributions of the orbital haloes deviates significantly from the dark matter, but can be approximated by an Einasto profile (Einasto 1965 thick green line). This density profile is given 
by

$\ln \left(\frac{n(r)}{n_{-2}}\right)=-\frac{2}{\alpha}\left(\left[\frac{r}{r_{-2}}\right]^{\alpha}-1\right)$,

where $n_{-2}$ and $r_{-2}$ are characteristic values of density and radius where the logarithmic slope, $d \ln n / d \ln r$, is equal to -2 . The third parameter, $\alpha$, controls the shape of the profile: $\alpha \approx 0.18$ yields one similar to the typical structure of dark matter haloes over the radial range resolved by cosmological simulations (e.g. Navarro et al. 2010); larger and smaller values correspond to profiles that are more or less curved than that of the dark matter, respectively.

Regardless of the mass of the primary, the spatial distribution of orbital haloes is well-approximated by eq. 2 with $\alpha \approx 1.2$ (shown as a thick green line in Figure 3), and a concentration parameter $c \equiv r_{200} / r_{-2} \approx 2.6$ (the stacked dark matter haloes have considerably higher concentrations, between 4.8 and 7.7), suggesting that they are significantly less concentrated than the dark matter. Nevertheless, their number density profiles do not show evidence of converging to a constant density core, but rather continue to rise all the way to the centre.

\subsection{Distribution of subhalo accretion times and apsis points}

In Figure 4 we plot the relative fraction of the number of apsis crossings for orbital haloes (green), orbital subhaloes (blue) and backsplash haloes (red), showing separately pericentres (left) and apocentres (right; note that only apocentres occurring after the initial turnaround radius are included). Shaded bars show results after combining all primary haloes, but there is little variation across the mass range covered by our sample. Solid and dotted open bars, for example, correspond to the lowest and highest host mass bins used in Figure 3

As anticipated in Figure 2 a large fraction of backsplash haloes, of order 63 per cent, are still approaching their first orbital apocentre, and the vast majority, $\approx 86$ per cent, have crossed pericentre only once. Only $\approx 5$ per cent have completed two or more apocentres. These numbers are even higher for orbital haloes: 76 per cent are still approaching first apocentre and $\gtrsim 95$ per cent have only one measured periapsis. This suggests that backsplash haloes and orbital haloes are either relatively recent arrivals, or occupy longperiod orbits about their primary haloes. Intriguingly, many orbital subhaloes must have also been accreted relatively recently: at $z=0$ as many as 30 per cent have yet to reach first apocentre, and about half have crossed pericentre only once. Less than $\approx 21$ per cent of orbital subhaloes have crossed pericentre three or more times; less than $\approx 13$ per cent have three or more measured apocentres. A significant fraction of orbital haloes, subhaloes and backsplash haloes are therefore unlikely to be well-mixed with the potential of their primary host haloes.

We explore this further in Figure 5, where we plot the distribution of subhalo accretion times for two separate bins of host mass. As expected, essentially all firstinfall subhaloes (red line) were accreted recently. The distribution peaks at $z=0$, and is largely confined to in-

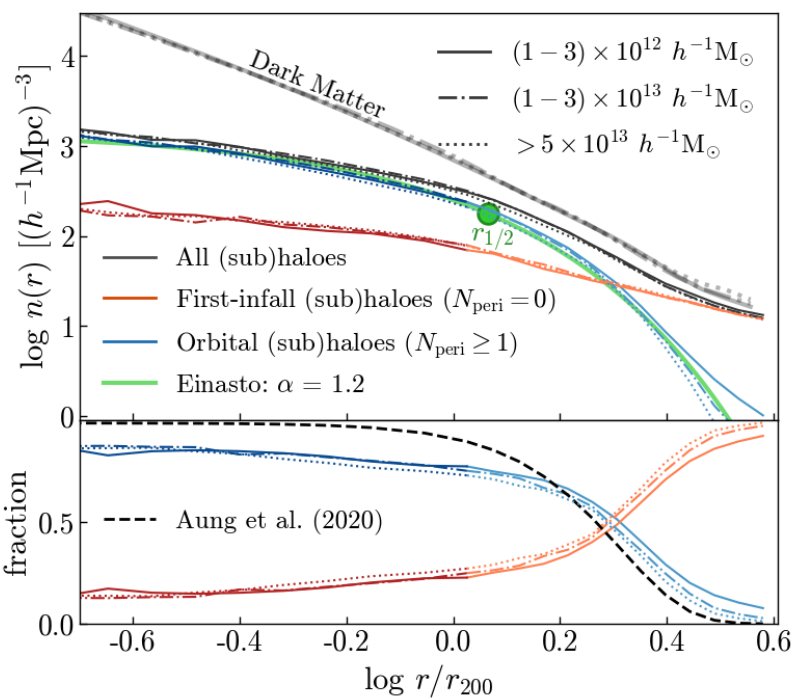

Figure 3. Spherically-averaged number density profiles of haloes and subhaloes surrounding primary hosts. Results are are shown for three (primary) mass bins: $(1-3) \times 10^{12} \mathrm{M}_{\odot}$ (solid lines; 1417 haloes), $(1-3) \times 10^{13} \mathrm{M}_{\odot}$ (dot-dashed; 211 haloes) and $\geqslant 5 \times 10^{13} \mathrm{M}_{\odot}$ (dotted; 37 haloes). Blue lines show the combined number density profiles of orbital subhaloes, orbital haloes and backsplash haloes; orange lines correspond to all first-infall haloes and subhaloes; black curves to the entire sample. The green line shows the best Einasto-fit to all three associated subhalo samples; the outsized circle marks the half-number radius of the fit, $r_{1 / 2}$, which exceeds $r_{200}$. Note that each sample, independently or together, differs substantially from the dark matter density distribution, which is shown using grey lines for each primary mass bin (normalised arbitrarily for comparison). Note also the smoothness of the combined distributions: they do not exhibit distinct features at $r_{200}$ that may be expected if that, or another arbitrary radius, were used as a classifier of "subhaloes" or "field haloes". As in Figure 2 we plot the fraction of haloes and subhaloes in each sample as a function of radius in the lower panel, along with the estimate of Aung et al. (2020) of the fraction of subhaloes that have completed at least one orbit.

fall times that do not exceed a half-crossing time ${ }^{1}$ defined $t_{\text {cross }} / 2=r_{200} / \mathrm{V}_{200}$ (shown as the right-most vertical grey line in Figure 5). Interestingly, orbital subhaloes (dark blue lines) have a strongly bimodal accretion time distribution: one peak clearly confined to infall times spanning $t_{\text {cross }} / 2 \leqslant$ $t_{\text {acc }} \leqslant t_{\text {cross }}$ (these are primarily subhaloes with $\mathrm{N}_{\text {apo }}=0$ ) and another peaking at $t_{\mathrm{acc}} \approx 3.5 \times t_{\text {cross }}$; the minimum between the two is reached at roughly $t_{\text {acc }} \approx 1.5 \times t_{\text {cross }}$. Not surprisingly, the minimum in the accretion time distribution for orbital subhaloes coincides with a maximum for backsplash haloes (light blue lines), since accreted material will typically require at least $t_{\text {cross }} \approx 1.8 \mathrm{Gyr}$ to exit the virial boundary of a halo once accreted. Indeed, the vast majority of backsplash haloes were accreted by their hosts at lookback times exceeding this timescale (second vertical grey line from the right).

For comparison, we also plot the distribution of infall times for "merged" subhaloes, i.e. those that have merged

1 A crossing, $t_{\text {cross }}=2 r_{200} / V_{200}$, time is roughly the time required for recently accreted material to reach orbital pericentre. 

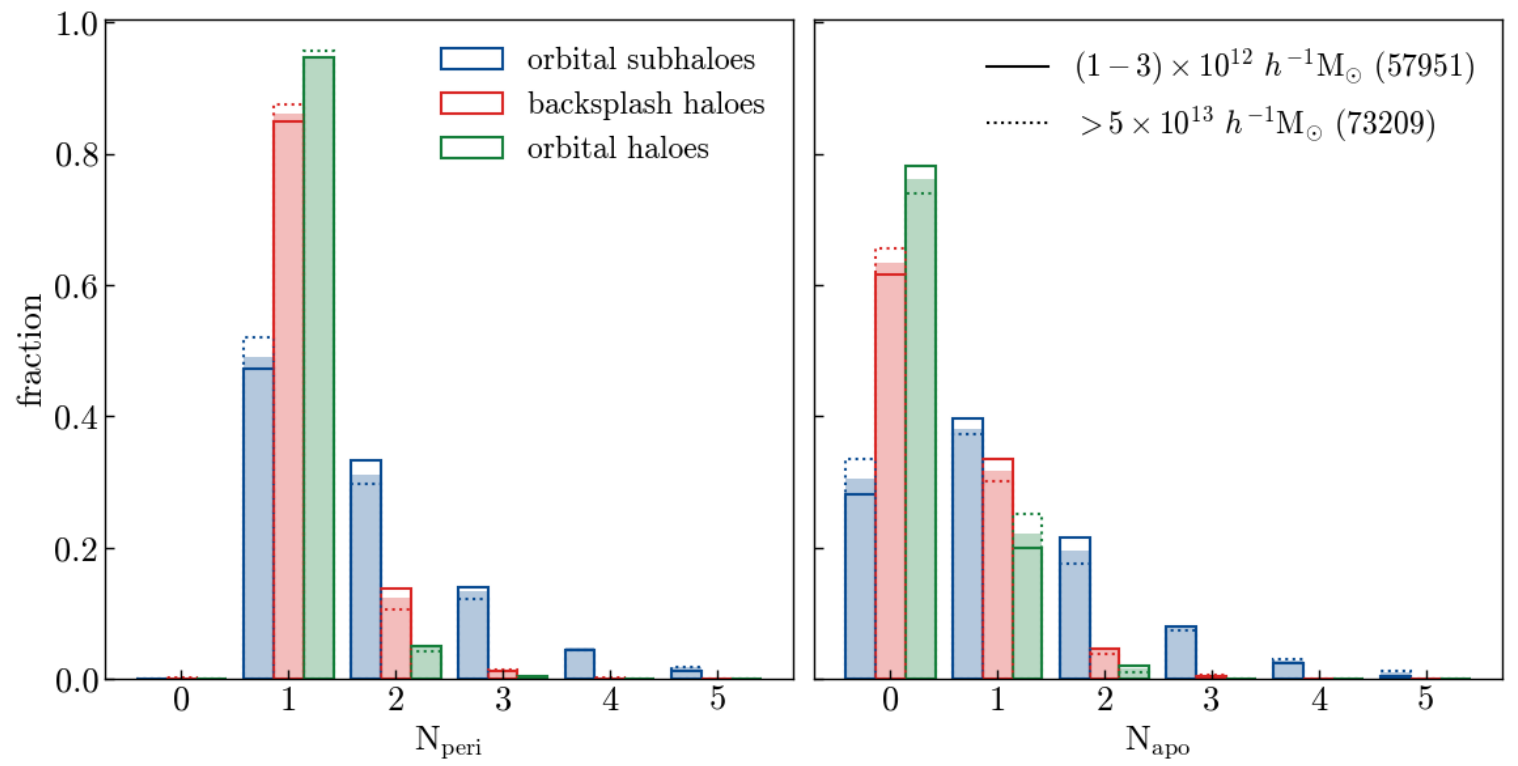

Figure 4. The fraction of orbital haloes and subhaloes, and backsplash haloes that have complete N pericentres (left) and apocentres (right; measured after their initial turnaround radius). As with previous figures, different colours differentiate orbital type: blue corresponds to orbital subhaloes, red to backsplash haloes, and green to orbital haloes. Shaded bars show results after stacking all primary haloes, regardless of mass; solid and dotted bars show results when only hosts of mass $(1-3) \times 10^{12} \mathrm{M}_{\odot}$ or $>5 \times 10^{13} \mathrm{M}_{\odot}$ are included, respectively.

with their host, were tidally disrupted or fell below our imposed 50-particle limit. The majority of these systems were accreted well before those that survive to the present day. For example, the peak accretion rate of merged subhaloes occurred around $z \approx 2.3$. Averaged over all hosts, we find that 95 per cent of subhaloes that survive to $z=0$ were accreted since $z=1.37$, whereas 95 per cent of those that do not, have accretion redshifts $z_{\text {acc }} \gtrsim 1.91$.

Overall, these results suggest that robustly measuring of the distribution of orbital apocentres directly from the orbital tracks of associated subhaloes may be challenging, since many have not yet had one. We demonstrate this in Figure 6, where we compare the current radial distribution of all associated subhaloes (black curves) to the distribution of their last measured apocentres ( $r_{\text {apo }}^{\text {last }}$ green lines). As noted above only $\approx 46$ per cent of associated subhaloes have at least one measured apocentre, and the majority of those that do not are backsplash haloes currently residing at radii $r \gtrsim r_{200}$. This explains why the radial distribution of associated subhaloes is broader than the distribution of their last-measured apocentres: haloes at very small radii are unlikely near apocentre, and so $r_{\text {sub }} \lesssim r_{\text {apo }}^{\text {last }}$, whereas many of those at large radii have not yet reached apocentre, and are not included in the plot. As we discuss below, this may have important implications for measuring the so-called "backsplash" radius of primary haloes by appealing to measurements of the apocentres of recently accreted material. This is an important caveat to consider, particularly if backsplash radii are to be used as observational probes of cosmology (e.g. Adhikari et al. 2018).

Nevertheless, it is possible to improve matters by estimating the orbital apocentres for associated subhaloes when one cannot be directly measured. Knowing the sphericallyaveraged potential of the host halo, $r_{\text {apo }}$ (occurring at $z \leqslant 0$ in these cases) can be estimated directly ${ }^{2}$ from the subhalo's orbital kinetic energy and angular momentum (see eq. 3.14 of Binney \& Tremaine 2008). Adding these predicted apocentres to the last-measured ones yields an apocentre distribution (blue lines) that peaks at $\approx 1.6 \times r_{200}$ (downward arrow), which coincides with the value for periodic orbits anticipated by the self-similar infall model of Bertschinger (1985). This is roughly 23 per cent larger than the peak of last-measured apocentres, occurring at $\approx 1.3 \times r_{200}$. Note also the extended tail of $r_{\text {apo }}$ : depending on primary mass, between roughly 10 to 13 per cent of associated subhaloes have $r_{\text {apo }} \gtrsim 3 \times r_{200}$, with between 1 to 4 per cent exceeding $5 \times r_{200}$. As discussed by Ludlow et al. (2009), many of these haloes $(\approx 37$ per cent in their study) occupy orbits that carry them beyond their initial turnaround radius, a scenario difficult to reconcile with simple analytic infall models.

We characterize the mass-dependence of the spatial distribution and apocentres of associated subhaloes quantitatively in Figure 77. From left to right, different panels correspond to the $(z=0)$ radii enclosing 50,75 and 95 per cent of all associated subhaloes (dashed purple lines), and their apocentres (solid orange line). For comparison, the solid black lines indicate $r_{200}$ and thick solid grey lines $r_{\text {edge }}=1.96 \times r_{200, \text { mean }}$, determined by Aung et al. (2020) to enclose roughly 99 per cent of associated subhaloes ${ }^{3}$ (note

2 We have verified the robustness of this procedure by comparing the last-measured apocentre to the predicted one for a sample of associated subhaloes with $\mathrm{N}_{\text {apo }} \geqslant 1$; the errors on the predicted $r_{\text {apo }}$ are typically random, and $\lesssim 10$ per cent.

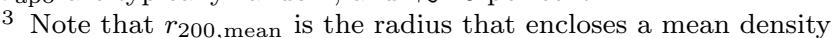
of $\Omega_{\mathrm{M}} \rho_{\text {crit }}$, which is slightly larger than $r_{200}$. For the purposes of Figure 7, we assume an NFW profile when converting $r_{200 \text {, mean to }}$ 

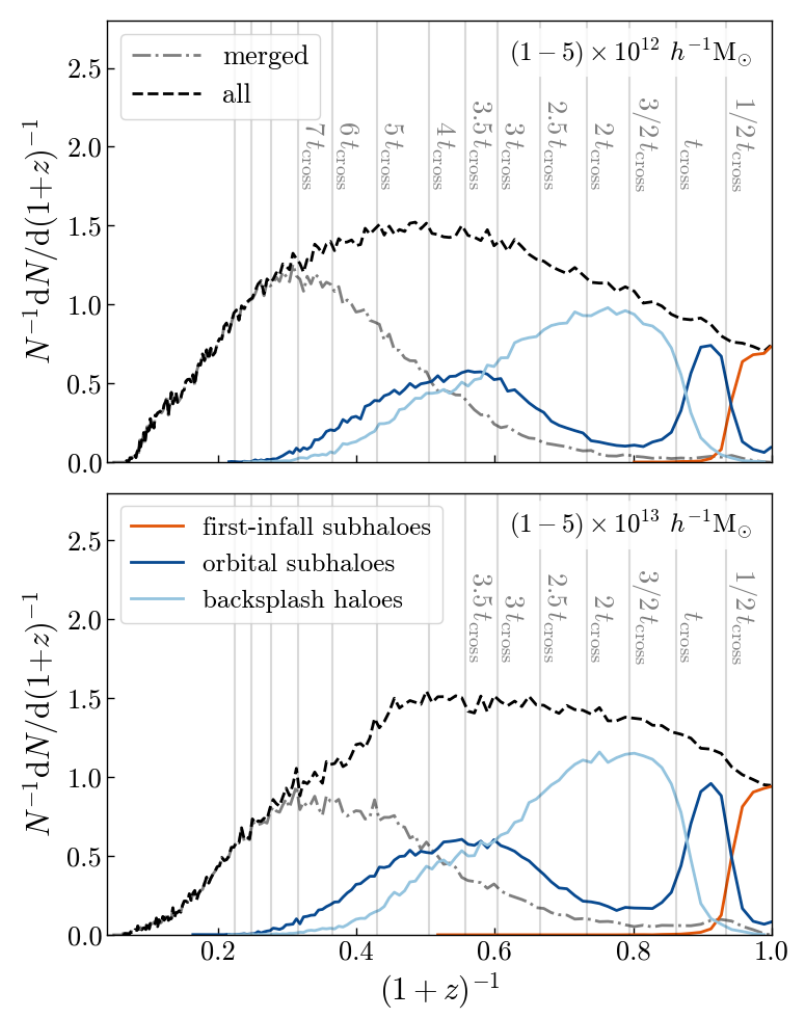

Figure 5. Distribution of subhalo accretion times for hosts in two separate mass bins (upper and lower panels). The red lines corresponds to first-infall subhaloes (i.e. $\mathrm{N}_{\text {peri }}=0$ and $r_{\text {sub }} \leqslant$ $r_{200}$ at $\left.z=0\right)$; dark blue lines to orbital subhaloes $\left(\mathrm{N}_{\text {peri }} \geqslant 1\right.$ and $r_{\mathrm{sub}} \leqslant r_{200}$ at $z=0$ ); light blue lines to backsplash haloes $\left(\mathrm{N}_{\text {peri }} \geqslant 1\right.$ and $r_{\text {sub }}>r_{200}$ at $\left.z=0\right)$. Dot-dashed grey lines show the distribution of accretion times for subhaloes that, by $z=0$, have either merged with their host haloes, were tidally disrupted, or fell below our 50-particle limit. The black dashed lines correspond to the entire subhalo population. Thin vertical lines mark integer multiples of half-crossing times, defined $t_{\text {cross }}=$ $2 r_{200} / \mathrm{V}_{200}$.

that these authors used the radial velocity distributions of haloes and subhaloes to distinguish infalling from accreted systems rather than explicitly tracking the orbits of individual objects). Thick dot-dashed grey lines enclose the equivalent fraction of splashback radii for dark matter particles (defined as the first apocentre after infall) determined from the fitting formulae of Diemer et al. (2017).

These measures of the spatial distribution of associated subhaloes differ in detail, but all paint a consistent picture: the spatial distribution extends far beyond $r_{200}$, and many associated subhaloes occupy orbits with unexpectedly large apocentres. For example, we find that only about half have apocentres that confine them to within $1.6 \times r_{200}$, and roughly 5 per cent have apocentres that exceed $3.4 \times r_{200}$. The radius enclosing a fraction $f_{\text {apo }}$ of all associated apocentres can be accurately described by the following fitting

$r_{200}$, and the thickness of the line indicates the variation expected for concentration parameters spanning $c=5$ to 15 .

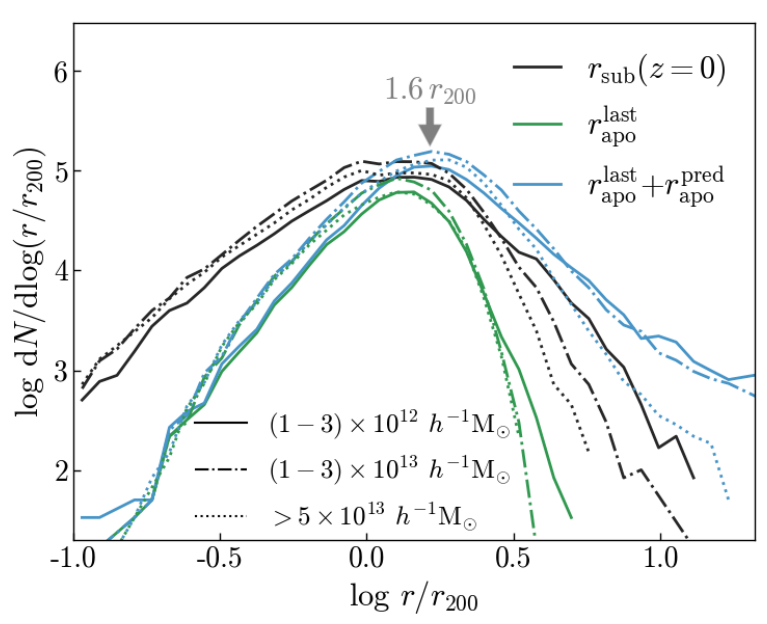

Figure 6. Distributions of associated subhalo radii, $r_{\text {sub }}(z=0)$ (black curves), last-measured apocentres, $r_{\text {apo }}^{\text {last }}$ (green lines), and the combined distribution of $r_{\text {apo }}^{\text {last }}$ and $r_{\text {apo }}^{\text {pred }}$ (blue lines), where the latter are the predicted apocentres for systems that have not yet had one (the predictions are based on the radial energy equation; see text for details). Results are shown for the same three mass bins used in previous figures: $(1-3) \times 10^{12} \mathrm{M}_{\odot}$ (solid), $(1-3) \times$ $10^{13} \mathrm{M}_{\odot}($ dot-dashed $)$ and $\geqslant 5 \times 10^{13} \mathrm{M}_{\odot}($ dotted $)$. Note that the distribution of last-measured apocentres is a poor indicator of the true apocentre distribution; this is primarily a result of the large fraction of associated subhaloes, around 54 per cent, that are (at $z=0)$ still approaching their first apocentre after turnaround.

formula:

$\log r\left(f_{\text {apo }}\right)=\log r_{200}+a \log f_{\text {apo }}+\left(1-f_{\text {apo }}\right)^{b}-c$

where $a=0.321, b=-0.089$ and $c=0.767$. Eq. 3, shown as a dotted green line in each panel of Figure 7 is valid for $0.05 \lesssim f_{\text {apo }} \lesssim 0.98$, and for host masses between $10^{12} \mathrm{M}_{\odot}$ and $10^{14} \mathrm{M}_{\odot}$.

Such extreme apocentres are not reached on their own, but require a considerable injection of orbital energy, likely through 3-body encounters with other associated subhaloes, or as a result of a group accretion event. We will return to this discussion in Section 3.5 but first turn our attention to the evolution of successive orbital apocentres.

\subsection{The evolution of orbital apocentres}

Figure 8 plots the ratio of successive apocentres, $r_{\text {apo }, i+1} / r_{\text {apo }, i}$, versus the subhalo-to-primary mass ratio at the initial one, $i$. Dots correspond to individual (associated) subhaloes; different lines mark the median relations for the entire sample of primaries (solid line), and for the highest (dotted; $\gtrsim 5 \times 10^{13} h^{-1} \mathrm{M}_{\odot}$ ) and lowest mass ones (dotdashed; $\left.\gtrsim(1-3) \times 10^{12} h^{-1} \mathrm{M}_{\odot}\right)$. The shaded regions show the $25^{t h}$ and $75^{t h}$ percentile scatter for the entire sample. Different panels correspond to more or less evolved orbits: the ratio of first apocentre to turnaround radius is shown in the top left panel, for example, and fourth-to-third apocentre ratio in the bottom-right. Dotted horizontal lines correspond to successive equal-amplitude apocentres. Subhaloes lying above or below these lines must have either gained or lost orbital energy along that segment of their orbit, respectively. 


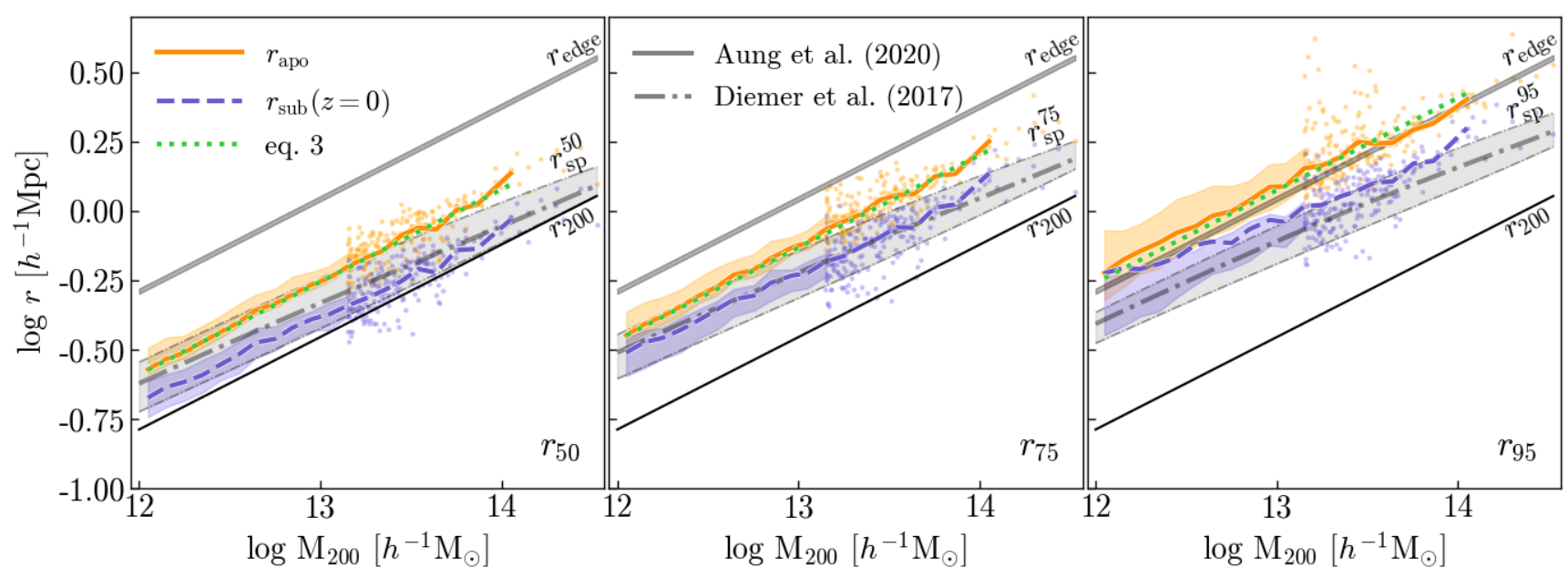

Figure 7. Radii that enclose 50, 75, and 95 per cent (left to right, respectively) of all associated subhaloes (blue dashed lines) and their apocentres (solid orange line) as a function of the virial mass of their primary. Lines correspond to the median trends measured after stacking all hosts in equally-spaced logarithmic mass bins of width $\Delta \log \mathrm{M}_{200}=0.1$; the shaded regions indicate the $25^{t h}$ and $75^{\text {th }}$ percentile scatter for masses below $1.4 \times 10^{13} h^{-1} \mathrm{M}_{\odot}$. Above this mass scale, where there are fewer than 40 haloes per bin, results for individual haloes are shown using coloured points. For comparison, we also show the radii (and corresponding scatter) enclosing the same fraction $f$ of dark matter particle splashback radii as thick grey lines (labelled $r_{\mathrm{sp}}^{f}$ in each panel), which were estimated using the empirical fit provided by Diemer et al. (2017). The thick grey line marked " $r$ edge" in each panel was determined by Aung et al. (2020) to enclose 99 per cent of associated subhaloes.

A few results are worth emphasizing. First, note that the majority of orbital energy loss occurs after first pericentric passage, i.e. between turn around and first orbital apocentre (top-left panel). On average, the ratio of first apocentre to the turnaround radius is $\approx 0.8$. Subsequent apocentres, at least for low mass subhaloes, tend to have comparable amplitudes, on average. This is indeed the trend expected from self-similar infall models, in which accreted material gradually loses energy, eventually reaching a stable orbit whose successive apocentres have equal amplitude. The arrows in each panel of Figure 8 for example, show the ratios anticipated by Bertschinger's (1985) model, which describes our simulation results reasonably wel

The effect, however, discussed in detail in van den Bosch et al. (2016), is mass dependent: subhaloes with masses exceeding $\gtrsim 0.01 \times \mathrm{M}_{200}$, for example, tend to experience an increased drain of orbital energy due to the increasing importance of dynamical friction; lower mass subhaloes behave as test particles in the potential of the host, and the massdependence of apocentre ratios weakens substantially. This effect ultimately leads to a spatial segregation of subhaloes according to their mass at infall, with more massive systems congregating toward the centres of haloes (see, e.g. Nagai \& Kravtsov 2005, Ludlow et al. 2009, van den Bosch et al. 2016).

Note also the considerable scatter along the ordinate axes of Figure 8 (the rms variance, $\sigma_{\text {apo }}$, is quoted in each panel). Indeed, as many as 11 (5.3) per cent of associated subhaloes have first apocentres that exceed their turnaround radius by as much as 25 (50) per cent, indicating a signifi-

4 We note that in the Bertschinger model the first apocentre of an accreted shell is of order 90 per cent of its turnaround radius, slightly larger than the results for the subhaloes plotted in the upper-left panel of Figure 8 cant gain in orbital energy after infall. Subhaloes may also lose energy after infall, and not always gradually, as would be expected from, e.g., dynamical friction or self-similar infall models. Roughly 42 (13) per cent have first apocentres that are at least 25 (50) per cent smaller than $r_{\text {ta }}$ (recall that, in Bertschinger's model, the first apocentre of an accreted shell has an apocentric distance $r_{\text {apo }} \approx 0.9 \times r_{\text {ta }}$ ). Although virtually all of the most massive accreted systems $\left(\mathrm{M}_{\text {sub }} \gtrsim 0.1 \times \mathrm{M}_{200}\right)$ experience a substantial reduction in orbital energy after infall, many low-mass systems - presumably unaffected by dynamical friction - do too. This unexpected result requires explanation. We will return to this point in Section 3.5

The outsized coloured points in Figure 8 show a few examples of subhaloes that illustrate these points; their orbits are shown in Figure 9. In each case, the loss or gain of orbital energy is invariably associated with an interaction. The upper-left panel, for example (marked as a yellow circle in Figure 8, shows a classic example of an "ejected" subhalo. Such extreme orbits are generated when accreted groups are tidally-dissolved by the main halo near their orbital pericentre, redistributing their orbital energy and often propelling low-mass members onto highly eccentric orbits. The remaining thin yellow lines show the orbits of other group members, which exhibit a broad range of first apocentres, from $r_{\text {apo }} \approx 0.6 r_{\mathrm{ta}}$ to $r_{\text {apo }} \approx 2.6 r_{\mathrm{ta}}$. At least two members of this group are placed on orbits so extreme that they are effectively removed from the system as a whole.

Of course, the opposite is also possible and energy can be abruptly lost during the tidal disruption of groups, and one such example is evident in the upper-left panel of Figure 9. Another, more extreme case is shown in the lower-left panel (green square in Figure 8). This subhalo is also a member of an accreted group (symbols along the orbital trajectories mark snapshots where each infalling halo was identified 
as a secondary subhalo of a more massive secondary halo), whose tidal disruption led to a rapid reduction in orbital energy; indeed, its first apocentre after infall is only of order $0.3 r_{\mathrm{ta}}$, and remains comparable thereafter.

While the types of interactions leading to abrupt changes in subhalo orbits are most common among members of infalling groups, the high number densities of subhaloes within $r_{200}$ means that penetrating encounter $\$^{5}$ between them, which may also perturb their orbits, should be relatively common (see e.g. Tormen et al. 1998, van den Bosch 2017). This, in principle, allows for less likely scenarios, such as the one plotted in upper-right panel of Figure 9 Here, the accreted halo loses orbital energy on first pericentre $\left(r_{\mathrm{apo}, 1} / r_{\mathrm{ta}} \approx 0.7\right)$ but regains even more on the second and is pushed to a third apocentre that exceeds it original turnaround radius $\left(r_{\mathrm{apo}, 2} / r_{\mathrm{ta}} \approx 1.2\right)$. Interestingly, this subhalo experienced a penetrating encounter with another close to its second orbital pericentre (triangles along the orbital trajectory indicate when this encounter occurred), leading to a 3-body encounter (the primary is the third body) favourable to the exchange of orbital energy and angular momentum.

It is also worth mentioning that instances of group accretion and penetrating encounters are not the only means of provoking unorthodox orbits such as these. In fact, any rapid change in the gravitational potential of the host which may be brought about by a merger, or any other large-scale fluctuation in the gravitational potential - can also lead to unexpected changes in the orbits of subhaloes as violent relaxation drives the system towards a new equilibrium.

While such unorthodox orbits are not difficult to identify, they are aberrant. Indeed, many subhaloes follow welldefined orbits that are not too dissimilar from those anticipated by simple spherical infall models. The purple curve in the lower-right panel (purple stars in Figure 9) shows a typical example of how subhalo orbits evolve. We will see below that the outliers in the distributions of apocentre ratios are mainly associated with systems that have been "preprocessed" by groups prior to infalling onto the primary halo.

\subsection{Pre-processing and the importance of group accretion}

A number of previous studies have identified pre-processing as an important regulator of galaxy evolution in dense environments. It may also have a significant impact on the evolution of the orbital trajectories. As discussed in the Introduction, pre-processing is naturally expected in the $\Lambda \mathrm{CDM}$ cosmology - or in any hierarchical model - due to the bottomup assembly of dark matter haloes. This motivates robustly quantifying the effect.

There are at least three ways in which a dark matter halo (or galaxy) may be pre-processed before being accreted

5 To estimate the frequency of penetrating encounters we first estimate the "size" of a subhalo as the virial radius $r_{200}^{\prime}$ corresponding to its $6 \mathrm{D}$ FOF mass. Encounters only occur after first infall, and are identified by tagging all subhaloes that cross another's $r_{200}^{\prime}$. by a primary halo. It may be a backsplash halo of the primary itself, and is again infalling. Having been previously accreted, these seemingly isolated systems have already passed through the much denser environment of the primary, potentially falling victim to tidal or ram pressure stripping. Similarly, it may be a subhalo or (secondary) backsplash halo of a infalling system. Cosmic web stripping is another possibility: large-scale cosmic filaments of gas and dark matter that feed primary haloes trace out large cosmological volumes, and can have large relative velocities with respect nearby haloes. Although of little importance for their dark matter content, haloes that cross path with these filaments may sustain losses of (gaseous) baryons as a result of ram pressure stripping (Benítez-Llambay et al. 2013).

As discussed above, roughly 59 per cent of associated subhaloes are currently beyond the virial radius of their host despite being bound to it. This adds a level of ambiguity to what is meant by "group accretion" as any infalling halo may also have its own population of secondary subhaloes and backsplash haloes. Because of this ambiguity, we identify the members of accreted groups in two ways: First, as the full population of (sub)haloes that were at any point prior to accretion by the primary identified as a secondary subhalo of a more massive infalling system (these systems have been "pre-processed" by secondary haloes but are not necessarily secondary subhaloes at the present time). And second, as the population of infalling systems that were identified as secondary subhaloes within a narrow radial window - which we initially take to be $(1-1.2) \times r_{200}-$ prior to their accretion by the host. The latter definition of group accretion is stricter, and demands that members of accreted groups be secondary subhaloes near the point of infall.

Figure 10 summarises the relative fraction (thick lines) of pre-processed haloes and subhaloes as a function of distance from their primary, showing separately those on firstinfall (i.e. $\mathrm{N}_{\text {peri }}=0$, left) and the combination of orbital subhaloes and backsplash haloes $\left(\mathrm{N}_{\text {peri }} \geqslant 1\right.$, right). As in several previous figures, results are shown for three separate primary mass bins, with mass increasing from top to bottom.

A large fraction of first-infall subhaloes $\left(r_{\text {sub }} \leqslant r_{200}\right.$, left panels) were substructures of more massive systems prior to their accretion by the primary - typically 40 to 55 per cent, depending on the primary's mass. Of these pre-processed haloes, roughly 31 to 38 per cent (depending on primary mass) were satellites at the time of accretion (i.e. were identified as secondary subhaloes at their point of entry into the primary; light shaded bars for $\left.r \leqslant r_{200}\right) ; \approx 29$ to 32 per cent are current secondary subhaloes of infalling haloes (dark shaded bars for $r>r_{200}$ ).

The right-hand panels of Figure 10 show the fraction of all orbital subhaloes and backsplash haloes at a given radial separation from their host that were pre-processed by a secondary halo prior to first infall (thick lines), or were accreted as part of a group (shaded regions). Pre-processing and group accretion are approximately equally important for systems already accreted (right panels), and for those currently infalling for the first time (left panels). These results depend only weakly on the mass of the primary, and on present-day radial separation.

In the upper panel of Figure 11 we plot the fraction of (sub)haloes of various orbital types as a function of their 

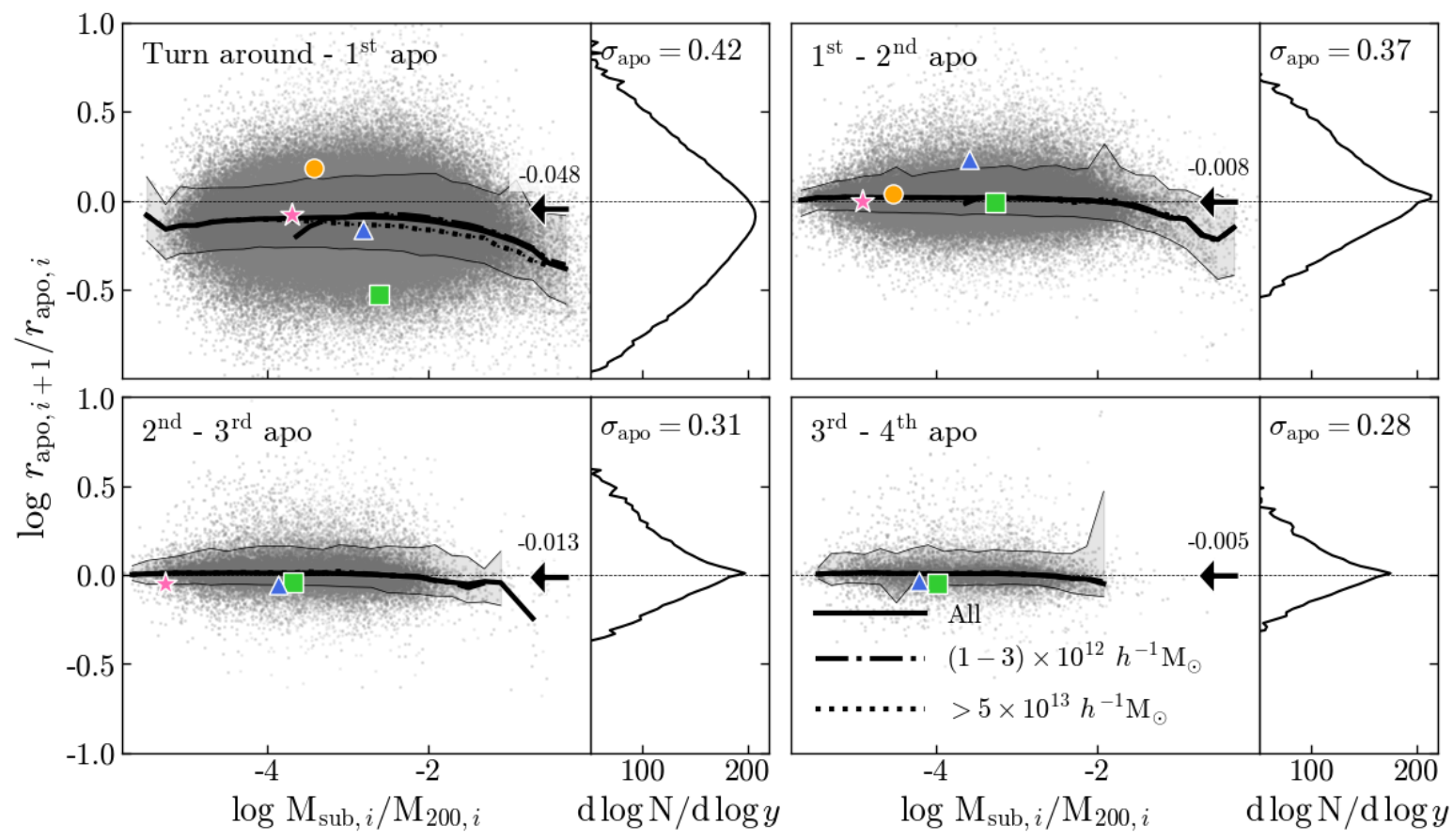

Figure 8. Ratio of successive apocentres for associated subhaloes plotted as a function of subhalo mass (expressed in units of the mass of the primary at apocentre $i$ ). From top-to-bottom, left-to-right panels correspond to the ratio of the first apocentre to the turnaround radius, $r_{\mathrm{ta}}$, then to the ratios of second-to-first, third-to-second, and fourth-to-third apocentres. In all panels, the mass of the subhalo and primary are measured at the first relevant apocentre (e.g. at the turnaround radius for those plotted in the upper-left panel). Note that only a small fraction, $\lesssim 1$ per cent, of all accreted haloes have $>4$ measurable apocentres and have been left out of the plot to avoid clutter. Individual (sub)haloes are shown using grey points, regardless of the mass of the primary; the thick black line and grey shaded region highlight the corresponding median and 25/75 percentile scatter. Medians for two separate primary mass bins, $(1-3) \times 10^{12} \mathrm{M}_{\odot}\left(\right.$ dot-dashed lines) and $\geqslant 5 \times 10^{13} \mathrm{M}_{\odot}$ (dotted lines), are also shown. The distributions are shown to the right $\left(\sigma_{\text {apo }}\right.$ in each side panel indicates the linear variance); the black arrows mark the values of $r_{\mathrm{apo}, i+1} / r_{\mathrm{apo}, i}$ anticipated by Bertschinger's (1985) spherically-symmetric self-similar infall model. Note that orbital energy loss is most pronounced between turnaround and first apocentre after accretion, and exhibits a relatively strong mass dependence for $\mathrm{M}_{\text {sub }} / \mathrm{M}_{200} \gtrsim 0.01$. Outsized coloured points mark specific subhaloes whose trajectories are plotted in Figure 9

mass at accretion time, normalized by the present-day mass of their primary host halo. We use different line styles for different primary mass bins, as in previous figures. Note the strong mass-dependence of the orbital subhalo and backsplash halo fractions, a result already hinted at in Figure 8 The mass dependence arises because the most massive associated subhaloes $\left(\mathrm{M}_{\text {acc }} \gtrsim 0.01 \times \mathrm{M}_{200}\right)$ tend to rapidly lose orbital energy due to dynamical friction after infall, and often remain orbital subhaloes until the present day.

As discussed in Ludlow et al. (2009), and as evident in Figure 11. low-mass members of accreted groups tend to be the ones "ejected" to higher energy orbits. This is because low-mass systems are more likely to be loosely bound to the group, and therefore to occupy large-amplitude orbits that are able to capture orbital energy when in phase with the orbit of the group within the primary. As a result, backsplash haloes tend to dominate the population of associated subhaloes below a characteristic mass that depends on the mass of the host.

Low-mass haloes are also more likely to be preprocessed than massive ones, as shown in the lower panel of Figure 11. For example, roughly half of all presentday subhaloes whose mass at accretion time is of order $\mathrm{M}_{\mathrm{acc}} \approx 10^{-3} \mathrm{M}_{200}$ were previously substructures of another (secondary) halo Even the most massive accreted systems are not entirely immune to the effects of pre-processing: for example, roughly 15 per cent of those with $\mathrm{M}_{\text {acc }} / \mathrm{M}_{200} \approx 0.1$ had been identified as subhaloes of other, more-massive systems prior to infall, and of order 5 per cent were substructures at the time of accretion.

\subsection{Impact of pre-processing and subhalo-subhalo encounters on the evolution of orbital trajectories}

We showed above (Figure 8) that successive apocentres of associated subhaloes evolve roughly as expected from simple spherical infall models, albeit with considerable variation among individual systems. The orbital trajectories of a few carefully-selected subhaloes, shown in Figure 9 suggest that the accretion of dynamically-associated groups of haloes, or close encounters between subhaloes, can lead to an exchange of orbital energy resulting in rather abrupt changes to their orbital apocentres. Do these particular examples encompass the majority of scenarios that may lead to deviant evolution of orbital apocentres? We explore this possibility in Figure 12

Consider first the upper-left panel, where we plot the 


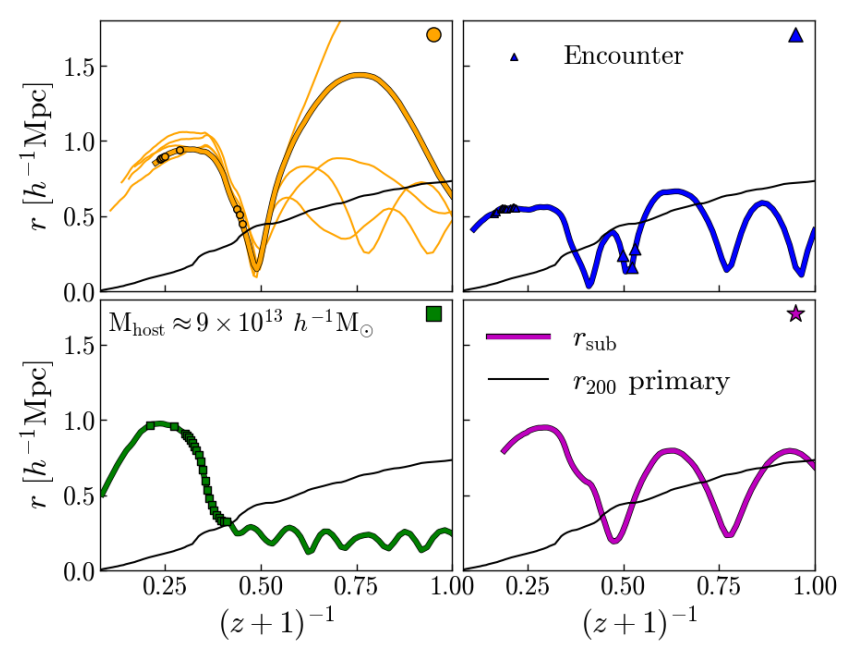

Figure 9. Orbital trajectories for the individual haloes and subhaloes highlighted in Figure 8 Identifying symbols, shown in the upper-right corner of each panel, are chosen to match those used in Figure 8 The upper-left panel shows an example of an extreme orbit (thick yellow line). This particular subhalo was accreted as part of a group (thin yellow lines correspond to orbits of other group members identified at the time of accretion) and was propelled onto a highly unorthodox orbit during the group's tidal dissociation near first pericentric passage. The dissociation of accreted groups not only ejects subhaloes along highly eccentric orbits, but can also lead to a rapid loss of orbital energy and angular momentum, resulting in some subhaloes becoming trapped on highly bound orbits, an example of which is shown in the lowerleft panel (note that snapshots in which these infalling haloes were identified as secondary subhaloes - i.e. subhaloes of an infalling secondary halo - are marked using symbols). Penetrating encounters between subhaloes, particularly near orbital pericentre, can also lead to energy exchange (upper-right panel). For comparison, we plot a "typical" orbital trajectory in the lower-right panel. The virial radius $r_{200}(z)$ of the primary halo is shown using a black line in all panels.

relative fraction of associated subhaloes in bins of $r_{\mathrm{apo}, 1} / r_{\mathrm{ta}}$ that were either a) pre-processed prior to accretion (grey line), b) accreted as part of a group of haloes (coloured lines), or c) as secondary haloes that were never identified as substructures of any other halo (solid black line). The differences are striking. For secondary haloes (c) the ratio of first apocentre to turnaround radius peaks at around $r_{\mathrm{apo}, 1} / r_{\mathrm{ta}} \approx 0.81$, comparable to analytic expectations. Very few of the outlier subhaloes are among this category. Instead, the outliers are primarily pre-processed haloes, i.e. haloes and subhaloes that are (or were) substructures of more massive systems prior to being accreted by the host. Indeed, pre-processed haloes account for more than two-thirds of systems with $r_{\text {apo,1 }} / r_{\text {ta }} \gtrsim 3$, and more than 80 per cent of those with $r_{\text {apo, } 1} / r_{\text {ta }} \lesssim 0.25$.

Many of these pre-processed haloes are members of loosely-bound groups that are tidally disrupted near first pericentre, giving rise to the unorthodox orbits seen in Figure 8 (this includes systems that both gain and loose orbital energy). This effect is portrayed more clearly by the coloured lines, which correspond to sub-samples of associated subhaloes that were pre-processed (i.e. identified as secondary subhaloes) within increasing radial apertures from

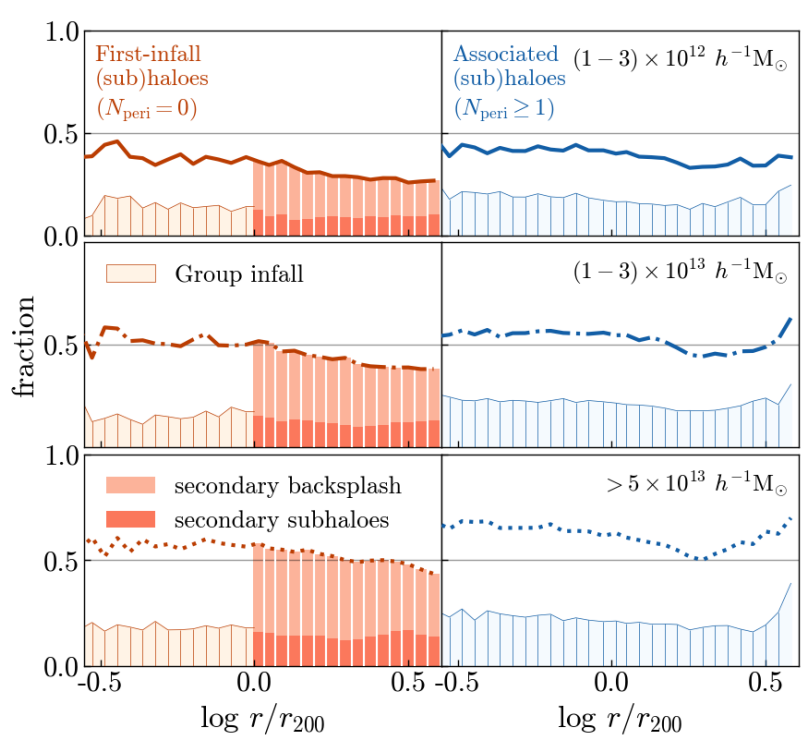

Figure 10. The relative importance of pre-processing for firstinfall haloes and subhaloes (left panels) and for orbital subhaloes and backsplash haloes (right panels) as a function of separation from primary haloes of different virial mass (top to bottom). Thick lines of different line-style show the fractions of (sub)haloes that were identified as substructures of a more massive system (excluding the primary halo) prior to infall or $z=0$, which ever comes first. First-infall haloes $\left(r_{\text {sub }}>r_{200}\right.$, left panels) are divided into secondary subhaloes (i.e. current subhaloes of secondary haloes; dark shaded region) and secondary backsplash haloes (light shaded region). First-infall subhaloes $\left(r_{\text {sub }} \leqslant r_{200}\right.$, left panels) are divided into those accreted in isolation (white regions) or as part of a larger group (light shaded region; see text for a definition of how group-infall events are classified). Similarly, the right-hand panels show the fraction of orbital subhaloes $\left(r_{\text {sub }} \leqslant r_{200}\right)$ and backsplash haloes $\left(r_{\text {sub }}>r_{200}\right)$ that were accreted in groups (shaded regions).

the host's centre prior to infall. The blue curve, for example, corresponds to systems that were formally accreted in a group (according to the definition of group accretion in section 3.4 , i.e. were pre-processed within a spherical aperture between 1 and $1.2 \times r_{200}$ prior to infall); these systems account for the majority of the most extreme cases. Orange and green curves, respectively, correspond to systems that were pre-processed within 1.5 and $2 \times r_{200}$ when on first approach. Increasing the aperture within which dynamicallyassociated infalling groups are identified clearly accounts for an increasing number of unexpectedly large and small first apocentres.

But what about subsequent orbits? The fact that many members of accreted groups are likely tidally dispersed on first pericentric passage suggests that whether a halo was pre-processed or not is unlikely to determine whether energy is lost or gained thereafter. This expectation is borne out by our simulations. The dashed grey lines in the remaining three panels, for example, show the ratio of subsequent apocentres (first-to-second to third-to-fourth) for systems that were pre-processed prior to first infall. In all cases this distribution remains approximately flat: evidently, preprocessing greatly affects the extreema of first apocentres, but not subsequent ones. 

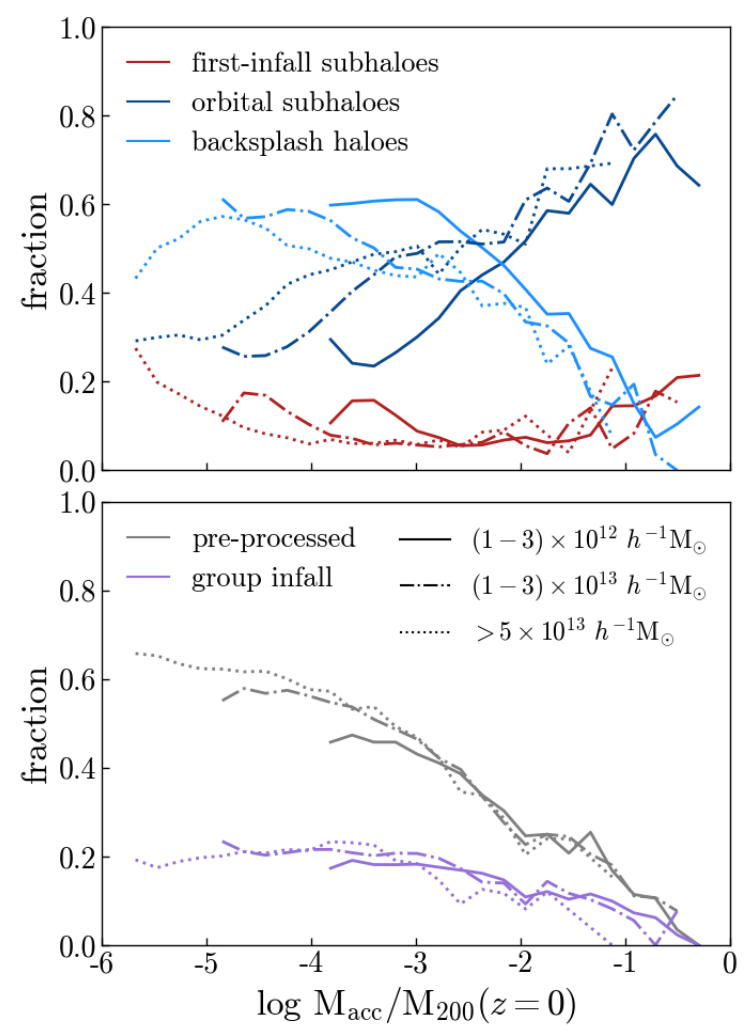

Figure 11. The fraction of haloes and subhaloes in various orbit categories as a function of their mass at the time of accretion, normalized by the present-day mass of their host halo. Upper panels show separately infalling subhaloes (i.e. $\mathrm{N}_{\text {peri }}=0$ and $r_{\text {sub }} \leqslant r_{200}$; red curves), orbital subhaloes $\left(\mathrm{N}_{\text {peri }} \geqslant 1\right.$ and $r_{\text {sub }} \leqslant r_{200}$; dark blue curves) and backsplash haloes (light blue); lower panels show the fractions of all haloes that were preprocessed prior to infall (grey curves), or were accreted as part of a larger group of haloes (purple lines). Different lines correspond to different host mass bins, as indicated.

As discussed in Section 3.3 (see also Tormen et al. 1998 van den Bosch 2017), penetrating encounters between subhaloes are common, typically affecting 60 per cent of all associated subhaloes at some point along their orbital paths. The majority of these encounters occur near pericentre - i.e. as close to the potential minimum of the host halo as their orbit allows - where subhalo number densities are highest, and therefore may constitute $\geqslant 3$-body interactions. Such 3 body interactions allow for a rapid redistribution of orbital energy between the interacting bodies and, as with infalling groups of haloes, can readily perturb orbits to higher or lower energies.

The remaining panels in Figure 12 show that penetrating encounters indeed affect the evolution of subsequent orbital apocentres. Each panel plots the relative fraction of haloes, in bins of $r_{\mathrm{apo}, i+1} / r_{\mathrm{apo}, i}$, that either experienced a penetrating encounter along that particular segment of their orbit (grey lines) or did not (black lines). In all cases, the extreema tend to be associated with those subhaloes that have experienced a penetrating encounter between $r_{\text {apo }, i}$ and $r_{\text {apo, } i+1}$; those that have not tend to follow more closely the evolution expected from simple spherical infall mod- els (the arrows in each panel mark the expectations from Bertschinger's model).

\subsection{The structure of first-infall and associated haloes and subhaloes}

It is well-established that the structure of dark matter haloes is largely determined by their collapse time (e.g. Bullock et al. 2001 Neto et al.|2007, Ludlow et al.|2013, 2014 |Correa et al. 2015), but also depends on environment (Avila-Reese et al. 2005), as well as on the various interaction histories haloes may have had with their neighbours (Li et al. 2013. Wang et al. 2020). Correlations between the structure of haloes and their varied assembly histories are often difficult to disentangle, but give rise to important secondary phenomena. One, known as assembly bias, quantifies the clustering strength of haloes as a function of their concentration or formation time (e.g. Gao et al. 2005): at fixed mass, older haloes cluster more strongly than younger ones, highly concentrated haloes more than less concentrated ones. A variety of interpretations have been put forth for the origin of the age-dependence of halo clustering, although it appears that no single process can explain it entirely (see, e.g., Mansfield \& Kravtsov 2020, for a recent discussion). For example, backsplash haloes have undergone tidal stripping by their hosts and appear older than field haloes of similar mass; they are also, by selection, more clustered due to their proximity to more massive hosts. Nevertheless, the assembly bias remains even after backsplash haloes have been accounted for (Wang et al. 2009b), suggesting that other dynamical processes are at work. Possibilities include the suppression of halo growth in dense environments due to tidal forces from large-scale structure (e.g. Hahn et al. 2009, Hearin et al. 2016), or large-scale tidal anisotropies (Paranjape et al. 2018).

In Figure 13 we quantify how the orbital histories of haloes and subhaloes affect their concentrations - a useful proxy for formation time - which we characterize using the magnitude and location of the maximum circular velocity, $V_{\max }$ and $R_{\max }$, respectively. This is a robust proxy for concentration for a couple of reasons: first, it can be estimated non-parametrically, and is therefore free from subtle biases that may be introduced when fitting to some suitablysmooth profile, such as NFW; second, while tidal stripping may substantially reduce the dark matter mass of a halo, its impact on $V_{\max }$ and $R_{\max }$ is much less dramatic (e.g. Gao et al. 2012).

The different panels of Figure 13 compare the $\mathrm{V}_{\max }-$ $R_{\text {max }}$ relations for the various samples of (sub)haloes to that of the entire population of haloes in the simulation (shown as a solid black line and repeated in all panels for comparison). Not surprisingly, the full population of (sub)haloes that lie within 2 virial radii of their hosts (dotted purple line, upperleft panel) are more concentrated than the average field halo, typically by about $\approx 15$ per cent (recall that roughly half of these are "associated" subhaloes and half are on first infall).

The upper-right and lower-left panels distinguish orbital (sub)haloes from those on first infall, respectively, and in each case the two samples have been subdivided into those inside and outside of $r_{200}$. Orbital subhaloes and backsplash haloes (solid and dashed blue lines in the upper-right panel) typically have concentrations that are of order 22 per 

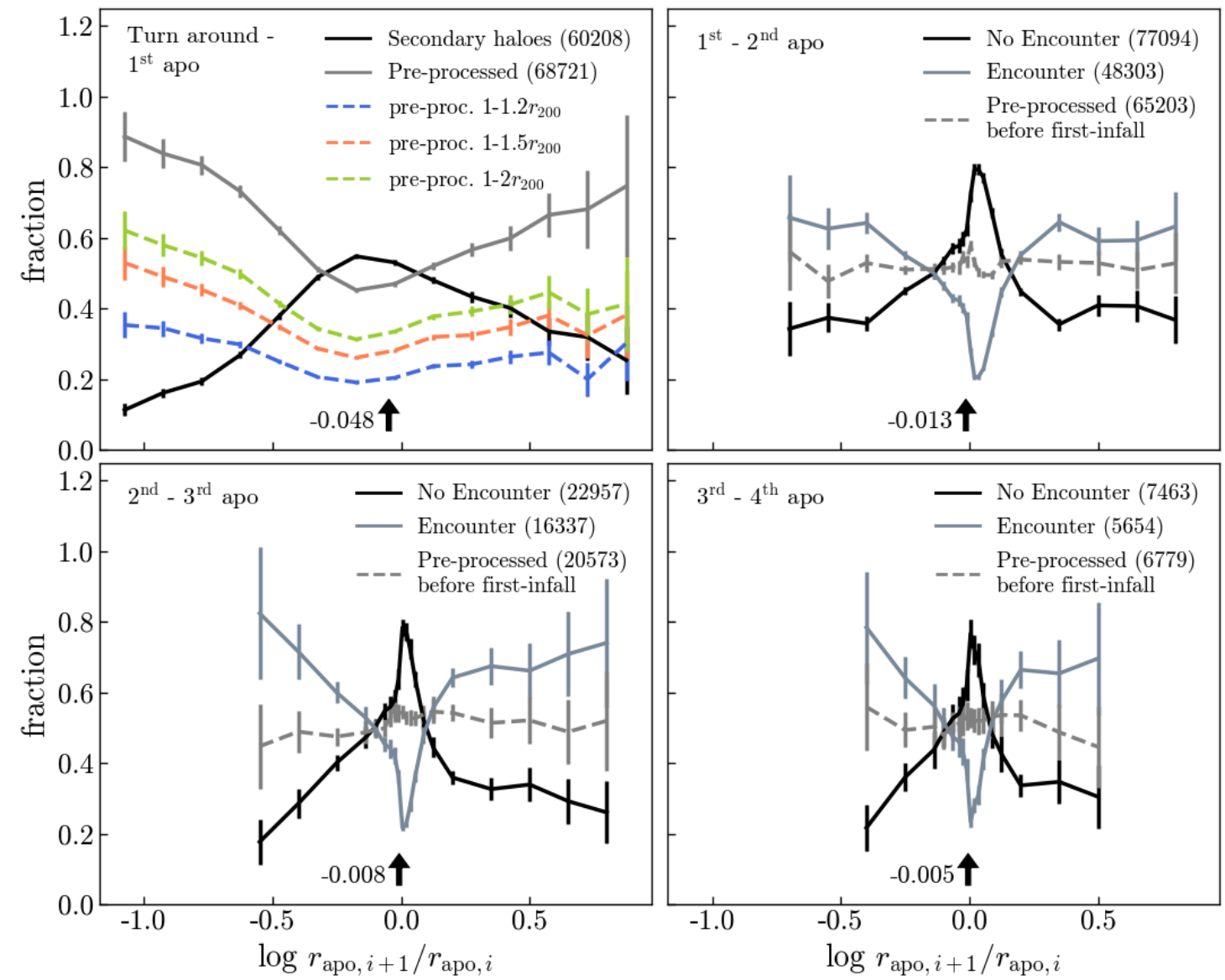

Figure 12. The fraction of associated subhaloes in bins of $\log r_{\mathrm{apo}, i+1} / r_{\mathrm{apo}, i}$, which quantifies the ratio of successive apocentric distances. As with Figure 8 different panels show less- or more-evolved orbits. The top-left panel, for example, corresponds to the ratio of the first apocentre (after infall) to the turnaround radius; the lower right panel to the ratio of the third-to-fourth apocentre. In the upper-left panel we divide subhaloes into two groups: one, shown using a solid black line, corresponds to pristine haloes (i.e. haloes that were never identified as substructures prior to infall), and another (grey line) showing those that were pre-processed before infall. Different coloured lines correspond to systems that were identified as secondary subhaloes within increasing radial apertures just prior to first accretion by the host (e.g. the blue line for systems that were tagged as secondary subhaloes between 1 to $1.2 \times r_{200}$; the green lines between 1 to $\left.2 \times r_{200}\right)$. Grey dashed lines in all other panels also correspond to haloes that were pre-processed prior to first infall, and solid grey lines to those that experienced a penetrating encounter with another subhalo between apocentre $i$ and $i+1$; solid black lines correspond to systems that did not encountered another subhalo along their orbital paths between the two relevant apocentres. Note that the extrema of these distributions are primarily associated with subhaloes that have interacted with others along their orbit paths.

cent higher than field haloes, except possibly for the highest $\mathrm{V}_{\max }$. Interestingly, infalling (sub)haloes have similar concentrations to field haloes - smaller by only a few per cent - regardless of their radial separation from the host (the dashed orange line, for example, corresponds to subhaloes that are on first infall, whereas the solid red line corresponds to infalling haloes at $\left.r>r_{200}\right)$.

The lower-right panel divides infalling haloes further, into "pristine" secondary haloes, i.e. those that have never been satellites of more massive systems (solid orange line), and pre-processed haloes that have (dashed green line; these are secondary backsplash haloes and secondary subhaloes). Secondary haloes on first infall have concentrations that are indistinguishable from those of field haloes, even when the former are restricted to radial separations $r \leqslant 2 \times r_{200}$; preprocessed haloes are, on average, $\approx 10$ per cent more concentrated. These results agree with the conclusions of $\mathrm{Li}$ et al.
(2013), who claim that tidal interactions between backsplash haloes and their former hosts are the primary mechanisms by which the concentrations of haloes in the environs of more massive systems are modified.

The systematic differences in the $\mathrm{V}_{\max }-\mathrm{R}_{\max }$ relations for haloes of different orbital type likely originate from their different tidal mass loss histories. We show this using "tidal tracks" (predicted by the model of Errani \& Navarro 2020. see also Hayashi et al. 2003; Peñarrubia et al. 2010. Green \& van den Bosch 2019), which describe the evolutionary paths followed by haloes in the $V_{\max }-R_{\max }$ plane as a function of the mass tidally stripped since infall. Curves are shown for three initial values of $\mathrm{V}_{\max }(50,150$ and $500 \mathrm{~km} / \mathrm{s})$ and start from the dashed line (the model of Ludlow et al. 2016). Symbols along each line mark remnant mass fractions corresponding to 0.5 (circle), 0.1 (triangle) and 0.01 (square). 

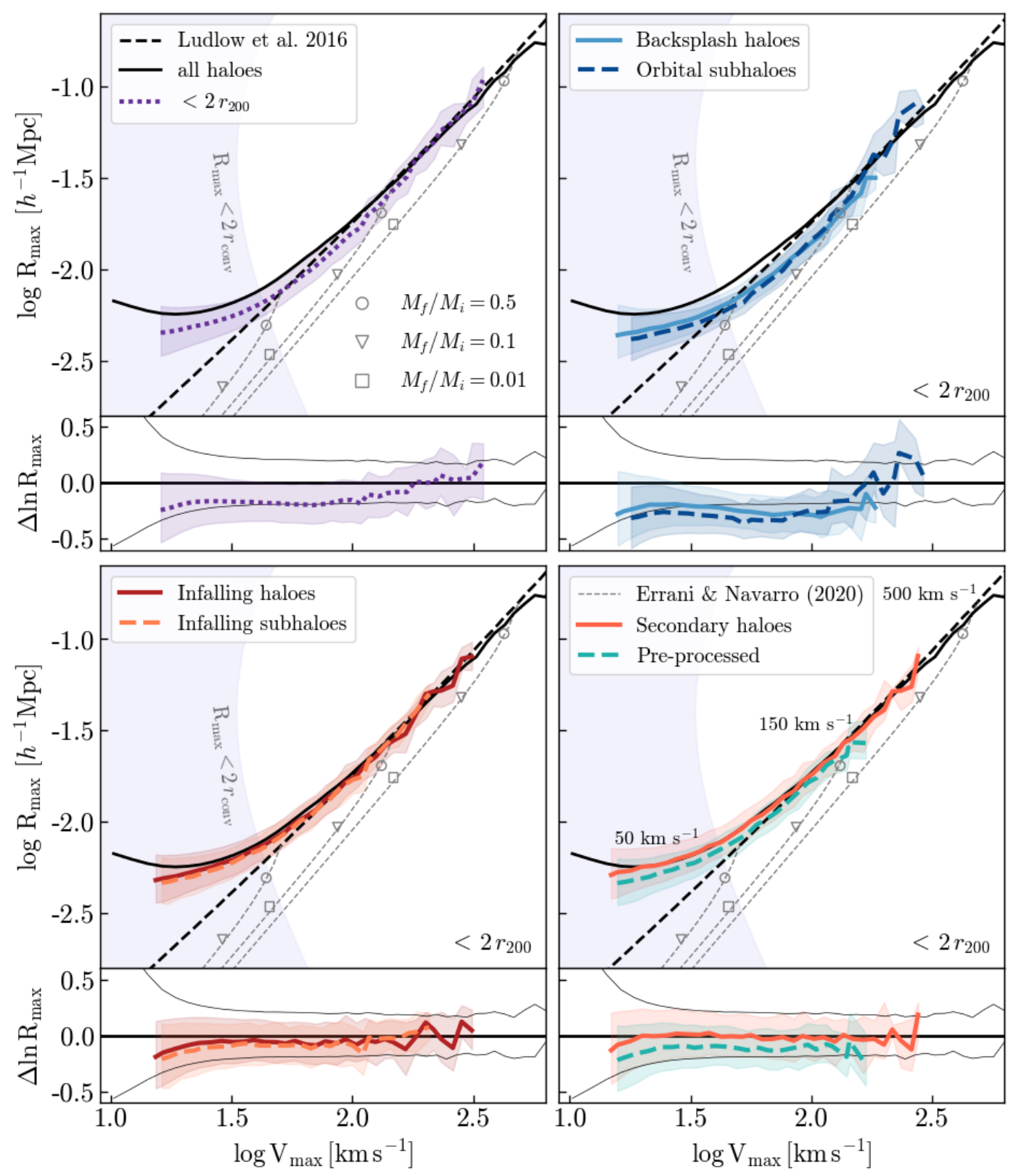

Figure 13. The $R_{\max }-V_{\max }$ relations for the various categories of haloes and subhaloes studied in this paper. The upper-left panel compiles results for all haloes and subhaloes that lie within 2 virial radii from their respective hosts (purple dotted line); the upper-right panel shows separately the median relations for all orbital subhaloes (i.e. $\mathrm{N}_{\text {peri }} \geqslant 1$ and $r_{\text {sub }} \leqslant r_{200}$; dashed blue lines) and backsplash haloes (i.e. $\mathrm{N}_{\text {peri }} \geqslant 1$ and $r_{\text {sub }}>r_{200}$; solid blue lines); the lower-left panel distinguishes infalling secondary haloes (solid red line) and secondary subhaloes (dashed orange line); the lower-right panel distinguishes pre-processed haloes (green dashed line) from isolated ones (solid orange). Note that, in all cases, we restrict the subhalo samples to those that lie within $2 \times r_{200}$ from the primary. The black solid line, repeated in all panels for comparison, shows the $R_{\max }-V_{\max }$ relation for all field haloes in the simulation volume (no isolation criteria are imposed). The dashed grey lines show the $R_{\max }-V_{\max }$ relation predicted by the analytic model of Ludlow et al. (2016). The curve delineating the open and shaded regions corresponds to the $R_{\max }-V_{\max }$ relation for a halo whose mass is determined by the constraint $\mathrm{R}_{\max }=2 \times r_{\text {conv }}$, where $r_{\text {conv }}$ is the convergence radius defined by Ludlow et al. (2019). Thin dashed lines show several "tidal tracks" predicted by the model of Errani \& Navarro (2020) for haloes with $V_{\max }=50,150$ and $500 \mathrm{~km} / \mathrm{s}$ at infall; the symbols along each of these curves indicate the surviving bound mass fraction.

\section{SUMMARY}

We used high-resolution, cosmological dark matter-only simulations to classify the orbital histories of haloes and subhaloes surrounding isolated hosts. Our analysis targets hosts that span the mass range $10^{12} \leqslant \mathrm{M}_{200} /\left[h^{-1} \mathrm{M}_{\odot}\right] \leqslant 3.4 \times 10^{14}$ $\left(8.6 \times 10^{4} \leqslant \mathrm{~N}_{200} \leqslant 2.9 \times 10^{7} ; 2309\right.$ in total $)$. As in previous work, we characterized the full population of haloes that ever crossed the virial radius of their host (so-called "associated" subhaloes) but considered, in addition, the remaining population that lie within four virial radii of the 
host's centre-of-potential. We carefully tracked the orbital trajectories for each of these haloes and subhaloes, tallying all apsis points in order to identify an unambiguous sample of "first-infall" haloes and subhaloes - i.e. those have that have not yet crossed pericentre on their orbit about the host - as well as "orbital" haloes and subhaloes - i.e. those that have completed at least one pericentric passage. By doing so, we were able to easily distinguish both haloes and subhaloes that are infalling for the first time from those that occupy more evolved orbits. Orbital systems are either "typical" substructure (i.e. have $r_{\text {sub }} \leqslant r_{200}$ at $z=0$ ), backsplash haloes $\left(r_{\text {sub }}>r_{200}\right.$ at $z=0$ but $r_{\text {sub }}<r_{200}$ in the past), or, less often, haloes whose pericentres occur outside their host's virial radius (referred to as "orbital" haloes). The variety of possible orbital trajectories considered in this paper are defined in Section 2.4 (see also Figure 1); their distribution in radial velocity-distance phase-space is shown in Figure 2.

In agreement with previous work (e.g. Diemand et al. 2004 Reed et al. 2005. Gao et al. 2004. Han et al. 2016), we find that the spatial distribution of associated subhaloes is substantially less concentrated towards the halo centre than the dark matter Their radial number density profiles, for example, can be well approximated by an Einasto profile with shape parameter $\alpha \approx 1.2$ (the concentration parameter is $c \approx 2.6$ ), implying a substantially shallower "cusp" than the traditional NFW profile. However, the number density of subhaloes does not approach a constant-density core, but rather increases (slowly) all the way to the halo centre (Figure 3). We find that half of all associated subhaloes are located within $\approx 1.24 \times r_{200}$ from the centre of their hosts, and $\approx 95$ per cent are located within $2.6 \times r_{200}$; associated systems make up 77 and 63 percent of all (sub)haloes within these radii, respectively.

We find that a large fraction of classically-defined (surviving) substructures (i.e. those with $r_{\text {sub }} \leqslant r_{200}$ ) were accreted very recently: roughly 15 per cent in the past halfcrossing time, $t_{\text {cross }} / 2=r_{200} / \mathrm{V}_{200} \approx 1.72 \mathrm{Gyr}$ (90 per cent of which are first-infall subhaloes), and 21 per cent in the interval $t_{\text {cross }} / 2 \leqslant t_{\text {acc }} \leqslant t_{\text {cross }}$ (primarily orbital subhaloes still approaching their first apocentre). Backsplash haloes of were primarily accreted at lookback times exceeding $t_{\text {cross }}$, which is the typical timescale required for recently-accreted material to first exit the virial boundary of its host halo. This suggests that many orbital subhaloes are recent arrivals: roughly half have crossed pericentre only once, and roughly one-third have yet to reach their first apocentre (after turnaround). Only $\approx 56$ per cent of all subhaloes within $r_{200}$ have completed at least one apocentric passage since infall (Figure 5).

Splashback radii are therefore not directly measurable from orbital tracks for many associated subhaloes, but may nevertheless be estimated provided their orbital energy and angular momentum is known. The ratio of consecutive apocentres (including both measured and predicted ones) for associated subhaloes with mass ratios $\mathrm{M}_{\text {sub }} / \mathrm{M}_{200} \lesssim 0.01$ approximately follow the pattern expected from simple selfsimilar infall models (e.g. Bertschinger 1985), although with slightly greater loss of orbital energy after first pericentre. Bertschinger's model, for example, predicts that the first apocentre of a typical orbit should be of order 90 per cent of its turn around radius; our simulations suggest a median value of $r_{\mathrm{apo}, 1} / r_{\mathrm{ta}} \approx 0.8$ (or a mean value of 0.87 ) for the lowest-mass subhaloes. Nevertheless, as expected from analytic models, the loss of orbital energy is most pronounced after first pericentric passage, after which the orbits of lowmass subhaloes typically reach a stationary state, and the ratio of successive apocentres approaches unity. These results do not apply to the orbits of the most massive subhaloes $\left(\mathrm{M}_{\text {sub }} / \mathrm{M}_{200} \gtrsim 0.01\right)$, which are repeatedly degraded due to dynamical friction (See Figure 8,)

Although these trends apply on average, the orbits of individual subhaloes exhibit a large variation between successive apocentres. For example, for hosts with $\mathrm{M}_{200} \approx$ $10^{12} h^{-1} \mathrm{M}_{\odot}\left(5 \times 10^{13} h^{-1} \mathrm{M}_{\odot}\right)$, roughly 26 (25) per cent of associated subhaloes have first apocentres that exceed their nominal turnaround radius, whereas $\approx 10$ (15) per cent have $r_{\mathrm{apo}, 1} / r_{\mathrm{ta}} \lesssim 0.5$. These outliers - common in cosmological haloes - deviate significantly from simple, analytic predictions. The discrepancy between orbits in simulations and those expected from analytic models appear to arise due to high-order interactions between subhaloes and the hosts in which they orbit. On first approach, the accretion of loosely-bound groups of haloes can account for the vast majority of outlier first apocentres (roughly twothirds of those with $r_{\text {apo, } 1} / r_{\text {ta }} \gtrsim 3.3$, and 80 per cent of those with $r_{\text {apo, } 1} / r_{\text {ta }} \lesssim 0.25$ were pre-processed prior to accretion). Rapid changes in orbital energy on subsequent orbits is mainly a result of penetrating encounters between subhaloes (see Figure 12 .

The orbital histories of (sub)haloes also affects their internal structure, results which we summarize in Figure 13. Infalling secondary haloes (i.e. those that have never been substructures of more massive haloes) follow a $V_{\max }-R_{\max }$ relation that is similar to the entire population of field haloes. Haloes that have been pre-processed, but not yet accreted by the host, are systematically more concentrated. Orbital subhaloes and backsplash haloes - which, by definition, have completed at least one pericentric passage about their host haloes - exhibit, relative to isolated field haloes, the most discrepant concentrations. These results imply that tidal encounters between associated subhaloes and their hosts are the primary mechanism by which the structural scaling relations of dark matter haloes are modified in dense environments.

\section{ACKNOWLEDGEMENTS}

We are grateful to our referee, Frank van den Bosch, for a useful report, which led to a number of improvements to our paper. We acknowledge various public PYTHON packages that have benefited our study: SCIPY (Jones et al. 2001), NUMPY (van der Walt et al. 2011), MATPLOTLIB (Hunter 2007) and IPYTHON (Pérez \& Granger 2007). ADL acknowledges financial support from the Australian Research Council through their Future Fellowship scheme (project number FT160100250). Parts of this research were conducted by the Australian Research Council Centre of Excellence for All Sky Astrophysics in 3 Dimensions (ASTRO 3D), through project number CE170100013. This research/project was undertaken with the assistance of resources and services from the National Computational Infrastructure (NCI), which is supported by the Australian Government, and supported by resources provided by the 
Pawsey Supercomputing Centre with funding from the Australian Government and the Government of Western Australia.

\section{REFERENCES}

Adhikari S., Sakstein J., Jain B., Dalal N., Li B., 2018, JCAP, 2018, 033

Aung H., Nagai D., Rozo E., Garcia R., 2020, arXiv eprints, p. arXiv:2003.11557

Avila-Reese V., Colín P., Gottlöber S., Firmani C., Maulbetsch C., 2005, ApJ, 634, 51

Bahé Y. M., McCarthy I. G., Balogh M. L., Font A. S., 2013, MNRAS, 430, 3017

Bahé Y. M., Schaye J., Barnes D. J., Dalla Vecchia C., Kay S. T., Bower R. G., Hoekstra H., McGee S. L., Theuns T., 2019, MNRAS, 485, 2287

Benítez-Llambay A., Navarro J. F., Abadi M. G., Gottlöber S., Yepes G., Hoffman Y., Steinmetz M., 2013, ApJL, 763, L41

Bertschinger E., 1985, ApJS, 58, 39

Bianconi M., Smith G. P., Haines C. P., McGee S. L., Finoguenov A., Egami E., 2018, MNRAS, 473, L79

Binney J., Tremaine S., 2008, Galactic Dynamics: Second Edition

Bullock J. S., Kolatt T. S., Sigad Y., Somerville R. S., Kravtsov A. V., Klypin A. A., Primack J. R., Dekel A., 2001, MNRAS, 321, 559

Correa C. A., Wyithe J. S. B., Schaye J., Duffy A. R., 2015, MNRAS, 452, 1217

Cortese L., Gavazzi G., Boselli A., Franzetti P., Kennicutt R. C., O'Neil K., Sakai S., 2006, A\&A, 453, 847

Crocce M., Pueblas S., Scoccimarro R., 2006, MNRAS, 373, 369

Davis M., Efstathiou G., Frenk C. S., White S. D. M., 1985, ApJ, 292, 371

De Lucia G., Kauffmann G., Springel V., White S. D. M., Lanzoni B., Stoehr F., Tormen G., Yoshida N., 2004, MNRAS, 348, 333

Diemand J., Moore B., Stadel J., 2004, MNRAS, 352, 535

Diemer B., 2017, ApJS, 231, 5

Diemer B., 2020, arXiv e-prints, p. arXiv:2007.10992

Diemer B., Kravtsov A. V., 2014, ApJ, 789, 1

Diemer B., Mansfield P., Kravtsov A. V., More S., 2017, ApJ, 843, 140

Einasto J., 1965, Trudy Astrofizicheskogo Instituta AlmaAta, 51, 87

Elahi P. J., Cañas R., Poulton R. J. J., Tobar R. J., Willis J. S., Lagos C. d. P., Power C., Robotham A. S. G., 2019, PASA, 36, e021

Elahi P. J., Poulton R. J. J., Tobar R. J., Cañas R., Lagos C. d. P., Power C., Robotham A. S. G., 2019, PASA, 36, e028

Elahi P. J., Welker C., Power C., Lagos C. d. P., Robotham A. S. G., Cañas R., Poulton R., 2018, MNRAS, 475, 5338 Errani R., Navarro J. F., 2020, arXiv e-prints, p. arXiv:2011.07077

Frenk C. S., White S. D. M., 2012, Annalen der Physik, 524,507

Gao L., Navarro J. F., Frenk C. S., Jenkins A., Springel V., White S. D. M., 2012, MNRAS, 425, 2169
Gao L., Springel V., White S. D. M., 2005, Mon. Not. Roy. Astron. Soc., 363, L66

Gao L., White S. D. M., Jenkins A., Stoehr F., Springel V., 2004, MNRAS, 355, 819

Garrison-Kimmel S., Boylan-Kolchin M., Bullock J. S., Lee K., 2014, MNRAS, 438, 2578

Ghigna S., Moore B., Governato F., Lake G., Quinn T., Stadel J., 1998, MNRAS, 300, 146

Gill S. P. D., Knebe A., Gibson B. K., 2005, MNRAS, 356, 1327

Gill S. P. D., Knebe A., Gibson B. K., Dopita M. A., 2004, MNRAS, 351, 410

Giocoli C., Tormen G., Sheth R. K., van den Bosch F. C., 2010, MNRAS, 404, 502

Green S. B., van den Bosch F. C., 2019, MNRAS, 490, 2091

Haggar R., Gray M. E., Pearce F. R., Knebe A., Cui W., Mostoghiu R., Yepes G., 2020, MNRAS, 492, 6074

Hahn O., Porciani C., Dekel A., Carollo C. M., 2009, MNRAS, 398, 1742

Han J., Cole S., Frenk C. S., Jing Y., 2016, MNRAS, 457, 1208

Han S., Smith R., Choi H., Cortese L., Catinella B., Contini E., Yi S. K., 2018, ApJ, 866, 78

Hayashi E., Navarro J. F., Taylor J. E., Stadel J., Quinn T., 2003, ApJ, 584, 541

Hearin A. P., Behroozi P. S., van den Bosch F. C., 2016, MNRAS, 461, 2135

Hou A., Parker L. C., Harris W. E., 2014, MNRAS, 442, 406

Hunter J. D., 2007, Computing In Science \& Engineering, 9, 90

Jones E., Oliphant T., Peterson P., et al.,, 2001, SciPy: Open source scientific tools for Python

Just et al. 2019, ApJ, 885, 6

Knebe A., Libeskind N. I., Knollmann S. R., MartinezVaquero L. A., Yepes G., Gottlöber S., Hoffman Y., 2011, MNRAS, 412, 529

Li R., Gao L., Xie L., Guo Q., 2013, MNRAS, 435, 3592

Li Y.-S., Helmi A., 2008, MNRAS, 385, 1365

Ludlow A. D., Bose S., Angulo R. E., Wang L., Hellwing W. A., Navarro J. F., Cole S., Frenk C. S., 2016, MNRAS, 460,1214

Ludlow A. D., Navarro J. F., Angulo R. E., Boylan-Kolchin M., Springel V., Frenk C., White S. D. M., 2014, MNRAS, 441, 378

Ludlow A. D., Navarro J. F., Boylan-Kolchin M., Bett P. E., Angulo R. E., Li M., White S. D. M., Frenk C., Springel V., 2013, MNRAS, 432, 1103

Ludlow A. D., Navarro J. F., Springel V., Jenkins A., Frenk C. S., Helmi A., 2009, ApJ, 692, 931

Ludlow A. D., Schaye J., Bower R., 2019, MNRAS, 488, 3663

Mansfield P., Kravtsov A. V., 2020, MNRAS, 493, 4763

Nagai D., Kravtsov A. V., 2005, ApJ, 618, 557

Navarro J. F., Ludlow A., Springel V., Wang J., Vogelsberger M., White S. D. M., Jenkins A., Frenk C. S., Helmi A., 2010, MNRAS, 402, 21

Neto A. F., Gao L., Bett P., Cole S., Navarro J. F., Frenk C. S., White S. D. M., Springel V., Jenkins A., 2007, MNRAS, 381, 1450

Paranjape A., Hahn O., Sheth R. K., 2018, MNRAS, 476, 3631 
Peñarrubia J., Benson A. J., Walker M. G., Gilmore G., McConnachie A. W., Mayer L., 2010, MNRAS, 406, 1290

Pérez F., Granger B. E., 2007, Computing in Science and Engineering, 9, 21

Planck Collaboration 2016, A\&A, 594, A13

Poole G. B., Mutch S. J., Croton D. J., Wyithe S., 2017, MNRAS, 472, 3659

Poulton R. J. J., Power C., Robotham A. S. G., Elahi P. J., 2019, MNRAS, p. 2798

Reed D., Governato F., Quinn T., Gardner J., Stadel J., Lake G., 2005, MNRAS, 359, 1537

Sales L. V., Navarro J. F., Abadi M. G., Steinmetz M., 2007a, MNRAS, 379, 1475

Sales L. V., Navarro J. F., Abadi M. G., Steinmetz M., 2007b, MNRAS, 379, 1464

Scoccimarro R., 1998, MNRAS, 299, 1097

Simpson et al. 2018, MNRAS, 478, 548

Springel V., 2005, MNRAS, 364, 1105

Springel V., Wang J., Vogelsberger M., Ludlow A., Jenkins A., Helmi A., Navarro J. F., Frenk C. S., White S. D. M., 2008, MNRAS, 391, 1685

Springel V., White S. D. M., Tormen G., Kauffmann G., 2001, MNRAS, 328, 726

Sunayama T., Hearin A. P., Padmanabhan N., Leauthaud A., 2016, MNRAS, 458, 1510

Tormen G., Diaferio A., Syer D., 1998, MNRAS, 299, 728

van den Bosch F. C., 2017, MNRAS, 468, 885

van den Bosch F. C., Jiang F., 2016, MNRAS, 458, 2870

van den Bosch F. C., Jiang F., Campbell D., Behroozi P., 2016, MNRAS, 455, 158

van der Walt S., Colbert S. C., Varoquaux G., 2011, CoRR, abs/1102.1523

Wang H., Mo H. J., Jing Y. P., 2009a, MNRAS, 396, 2249

Wang H., Mo H. J., Jing Y. P., 2009b, MNRAS, 396, 2249

Wang K., Mao Y.-Y., Zentner A. R., Lange J. U., van den Bosch F. C., Wechsler R. H., 2020, arXiv e-prints, p. arXiv:2004.13732

Wetzel A. R., Deason A. J., Garrison-Kimmel S., 2015, ApJ, 807, 49

Wetzel A. R., Tinker J. L., Conroy C., van den Bosch F. C., 2014, MNRAS, 439, 2687

White S. D. M., Rees M. J., 1978, MNRAS, 183, 341

Yang X., Mo H. J., van den Bosch F. C., Pasquali A., Li C., Barden M., 2007, ApJ, 671, 153 